Swarthmore College

Works

6-4-2018

\title{
Multiple Stellar Flybys Sculpting The Circumstellar Architecture In RW Aurigae
}

\author{
J. E. Rodriguez \\ R. Loomis \\ S. Cabrit \\ T. H. Haworth \\ S. Facchini
}

See next page for additional authors

Follow this and additional works at: https://works.swarthmore.edu/fac-physics

Part of the Astrophysics and Astronomy Commons

Let us know how access to these works benefits you

\section{Recommended Citation}

J. E. Rodriguez, R. Loomis, S. Cabrit, T. H. Haworth, S. Facchini, C. Dougados, R. A. Booth, Eric L.N. Jensen, C. J. Clarke, K. G. Stassun, W. R. F. Dent, and J. Pety. (2018). "Multiple Stellar Flybys Sculpting The Circumstellar Architecture In RW Aurigae". Astrophysical Journal. Volume 859, Issue 2. DOI: 10.3847/ 1538-4357/aac08f

https://works.swarthmore.edu/fac-physics/357

This work is brought to you for free by Swarthmore College Libraries' Works. It has been accepted for inclusion in Physics \& Astronomy Faculty Works by an authorized administrator of Works. For more information, please contact myworks@swarthmore.edu. 


\section{Authors}

J. E. Rodriguez, R. Loomis, S. Cabrit, T. H. Haworth, S. Facchini, C. Dougados, R. A. Booth, Eric L.N. Jensen, C. J. Clarke, K. G. Stassun, W. R. F. Dent, and J. Pety 


\title{
Multiple Stellar Flybys Sculpting the Circumstellar Architecture in RW Aurigae
}

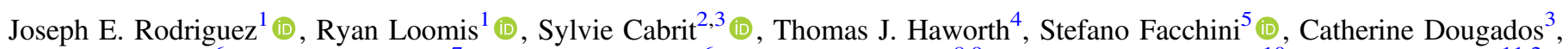 \\ Richard A. Booth $^{6}$, Eric L. N. Jensen ${ }^{7}$ (i), Cathie J. Clarke ${ }^{6}$, Keivan G. Stassun ${ }^{8,9}$ (i), William R. F. Dent ${ }^{10}$, and Jérôme Pety ${ }^{11,2}$ (i) \\ ${ }^{1}$ Harvard-Smithsonian Center for Astrophysics, 60 Garden Street, Cambridge, MA 02138, USA \\ ${ }^{2}$ Sorbonne Université, Observatoire de Paris, Université PSL, CNRS, LERMA, F-75014 Paris, France \\ ${ }^{3}$ Univ. Grenoble Alpes, CNRS, IPAG, F-38000 Grenoble, France \\ ${ }^{4}$ Astrophysics Group, Imperial College London, Blackett Laboratory, Prince Consort Road, London SW7 2AZ, UK \\ ${ }^{5}$ Max-Planck-Institut für Extraterrestrische Physik, Giessenbachstraße 1, D-85748 Garching, Germany \\ ${ }^{6}$ Institute of Astronomy, University of Cambridge, Madingley Road, Cambridge CB3 OHA, UK \\ ${ }_{8}^{7}$ Department of Physics and Astronomy, Swarthmore College, Swarthmore, PA 19081, USA \\ ${ }^{8}$ Department of Physics and Astronomy, Vanderbilt University, Nashville, TN 37235, USA \\ ${ }^{9}$ Department of Physics, Fisk University, Nashville, TN 37208, USA \\ ${ }^{10}$ Joint ALMA Observatory, Alonso de Cordova 3107, 763-0355 Vitacura, Santiago, Chile \\ ${ }^{11}$ I.R.A.M., 300 rue de la Piscine, Domaine Universitaire, F-38406 Saint Martin d'Hères, France \\ Received 2018 March 28; revised 2018 April 20; accepted 2018 April 24; published 2018 June 4
}

\begin{abstract}
We present high-resolution ALMA Band 6 and 7 observations of the tidally disrupted protoplanetary disks of the RW Aurigae binary. Our observations reveal tidal streams in addition to the previously observed tidal arm around RW Aur A. The observed configuration of tidal streams surrounding RW Aur A and B is incompatible with a single star-disk tidal encounter, suggesting that the RW Aurigae system has undergone multiple flyby interactions. We also resolve the circumstellar disks around RW Aur A and B, with CO radii of 58 au and 38 au consistent with tidal truncation, and 2.5 times smaller dust emission radii. The disks appear misaligned by $12^{\circ}$ or $57^{\circ}$. Using new photometric observations from the American Association of Variable Star Observers (AAVSO) and the All Sky Automated Survey for SuperNovae (ASAS-SN) archives, we have also identified an additional dimming event of the primary that began in late 2017 and is currently ongoing. With over a century of photometric observations, we are beginning to explore the same spatial scales as ALMA.
\end{abstract}

Key words: binaries: general - protoplanetary disks - stars: individual (RW Aur)

Supporting material: data behind figures

\section{Introduction}

The evolution of the circumstellar environment of a $\mathrm{T}$ Tauri star (TTS) from gas and dust to planets involves complex dynamical processes that are directly influenced by the presence of companions. It is known that most TTSs are in binaries (Ghez et al. 1993; Leinert et al. 1993; Richichi et al. 1994; Simon et al. 1995; Ghez et al. 1997), and the process of planet formation can be altered and disrupted by a stellar companion that gravitationally influences the gas and dust within the star's circumstellar disk. Specifically, strong binary interactions will stir up the disk, enhancing planetesimal collisions. Additionally, binary interactions can excite the orbital eccentricities and inclinations of planetesimals, as shown by stellar flyby models to explain the outer structure of the the Kuiper Belt in our own solar system (Ida et al. 2000). Theoretical models predict that young binary systems may truncate the surrounding circumstellar material at a distance of up to three times the orbital separation of the two stars (Artymowicz \& Lubow 1994). This might explain the deficiency of debris disks around main-sequence binaries with separations of between 3 and 50 au when compared to other single star systems and other binaries (Trilling et al. 2007).

A demonstration of the impact of binary interactions on disk evolution (and possibly planet formation) is the classical TTS RW Aurigae. The RW Aur system is $140 \mathrm{pc}$ away (van Leeuwen 2007) and comprised of at least two stellar objects, RW Aur A and B, which are separated by $\sim 1$ "' 5 (Herbig \& Bell 1988; Duchêne et al. 1999; Stout-Batalha et al. 2000). RW Aur A has a large bipolar jet extending out to $\sim 100^{\prime \prime}$ and containing many emission knots (Mundt \& Eislöffel 1998; López-Martín et al. 2003). Using the Plateau de Bure Interferometer (PdBI), this system was mapped in ${ }^{12} \mathrm{CO}$ and dust continuum by Cabrit et al. (2006) down to a resolution of 0 " $9 \times 0$ " 6 . Several unusual features were detected in these observations: a very compact rotating disk around RW Aur A, a large $\sim 600$ au long ${ }^{12} \mathrm{CO}$ tidal arm stretching from it, and a circumstellar structure with complex kinematics around RW Aur B. Based on a comparison with early numerical simulations by Clarke \& Pringle (1993), Cabrit et al. (2006) proposed that RW Aur B recently had a close encounter with RW Aur A, tidally stripping the original circumstellar disk around A. Although the disk around RW Aur A was not spatially resolved, comparison of its $\mathrm{CO}$ profile with theoretical models for Keplerian disks (Beckwith \& Sargent 1993) indicated a small radius of $40-57 \mathrm{au}$, inclined $45^{\circ}-60^{\circ}$ to the line of sight. It was also speculated that the eccentric flyby of RW Aur B possibly contributed to a temporary enhancement of the disk accretion rate onto RW Aur A $\left(4 \times 10^{-8}\right.$ $-2 \times 10^{-7} M_{\odot} \mathrm{yr}^{-1}$, Hartigan et al. 1995; Facchini et al. 2016). Whether the A disk will survive this enhanced accretion episode is unclear: at the current rate, and with the disk mass estimated from dust $\left(3 \times 10^{-4} M_{\odot}\right.$ Andrews \& Williams 2005), the disk would be accreted in only 1000 years.

To add to the mystery, RW Aur has also been exhibiting strong occultation events. After being photometrically monitored for over a century (Beck \& Simon 2001), the RW Aur system faded by $\sim 2$ mag in late 2010 for 180 days (Rodriguez et al. 2013). From analyzing over 110 years of $B$-band 
photometric observations (photoelectric, photographic, and visual observations), no event similar in duration and depth was seen, but the nominal brightness of RW Aur does vary on decade timescales (Berdnikov et al. 2017). Using basic kinematics, it was determined that the occulting object was moving at a few $\mathrm{km} \mathrm{s}^{-1}$ and was likely $\sim 0.3$ au in diameter. Three years later, another large dimming ( $>4.5 \mathrm{mag})$ occurred, lasting 2 years (Petrov et al. 2015; Rodriguez et al. 2016; Lamzin et al. 2017). Near-infrared (near-IR) observations of the RW Aurigae system suggest that the occulting body consists of large grains $(\geqslant 1 \mu \mathrm{m})$, causing gray absorption (Schneider et al. 2015). During the more recent dimming, there was an observed excess in the near-IR ( $L$ and $M$ ) attributed to hot dust $(\sim 1000 \mathrm{~K})$ in the inner disk (Shenavrin et al. 2015). VRI polarimetry observations during the 2014-2016 dimming show a $20 \%-30 \%$ increase in polarization, consistent with what has been seen for UX Ori stars (Lamzin et al. 2017). The high accretion rate of RW Aur A remained constant during both dimming events (Chou et al. 2013; Shenavrin et al. 2015). The cause of these unexpected dimming events is likely an occultation by a dust screen, but the origin of this screen is unclear and debated (Rodriguez et al. 2013; Petrov et al. 2015; Shenavrin et al. 2015; Bozhinova et al. 2016; Facchini et al. 2016; Berdnikov et al. 2017).

To further investigate the possibility that the unusual morphology of RW Aur might be explained by a tidal encounter in the form of a star-disk flyby, Dai et al. (2015) used a series of hydrodynamic ( $\mathrm{SPH}$ ) simulations to identify the orbital parameters that best reproduce all the features of RW Aur inferred from the PdBI observations. This included the length of the tidal arm, separation between RW Aur A and B, disk position angles, and relative stellar proper motions found by Bisikalo et al. (2012). Furthermore, they post-processed the hydrodynamic simulations with the non-local thermal equilibrium (non-LTE) radiative transfer code TORUS (Harries 2000; Rundle et al. 2010) to compute synthetic molecular line and dust continuum observations to compare with the observations from Cabrit et al. (2006), finding excellent agreement with the $\mathrm{CO}$ optical depths, kinematic signatures in the line profiles, and observed continuum and $\mathrm{CO}$ flux densities, although the $\mathrm{CO}$ emission around B is slightly underestimated. The model also predicts that the line of sight to RW Aur A currently intersects a bridge of stripped-off material between the two stars. Although of low column density $\left(N_{\mathrm{H}} \leqslant 10^{-4} \mathrm{~g} \mathrm{~cm}^{-2}\right.$, i.e., $A_{V} \leqslant 0.1 \mathrm{mag}$ ), it was argued that the bridge structure may have small clumps of denser material that may be able to occasionally dim the central star (Dai et al. 2015).

In this paper, we present new Atacama Large Millimeter/ submillimeter Array (ALMA) observations of the RW Aurigae system showing the aftermath of the star-disk interaction at high angular resolution. The new observations show additional tidal streams, suggesting the occurrence of multiple fly-bys of RW Aur B. Using new photometric observations by the American Association of Variable Star Observers (AAVSO) and the All Sky Automated Survey for SuperNovae (ASASSN), we analyze the full 2014-2016, 2016-2017, and the currently ongoing 2017-2018 dimming events. We also present an analysis of the archival AAVSO observations prior to 1960, showing a large dimming event that occurred in the late 1930s. Last, we announce that after a short period of quiescence after the 2016-2017 dimming, RW Aur began to fade again in 2017 mid-November. These new observations help to shed light on the intriguing nature of RW Aur itself, as well as on the more general links between disk evolution and planet formation in binary systems.

The paper is organized in the following way: our ALMA and photometric observations are presented in Section 2. Our analysis of the high-resolution ALMA data is discussed in Section 3. We discuss all six observed dimming events and the derived kinematics in Section 4. In Section 5 we explore the impact these new results have on our understanding of the RW Aurigae system. Our results and conclusions are summarized in Section 6.

\section{Observations}

\subsection{ALMA}

As part of ALMA Cycle 3 and 4 projects 2015.1.01506.S and 2016.1.00877.S, RW Aur was observed in Band 6 (225 GHz) with the $12 \mathrm{~m}$ array on UT 2016 September 29/ 30 and UT 2016 December 07/08 for a total integration time of 311.17 minutes. The observations included 39 antennas with a baseline of $15-3248 \mathrm{~m}$. The quasar J0510+1800 was used as the flux, phase, and bandpass calibrator for all scheduling blocks except one, which used J0512+2927 as the phase calibrator. In addition, Band 6 observations were taken with the $7 \mathrm{~m}$ array for a total additional integration time of 217.23 minutes. This observation used 11 antennas with a baseline range of 9-45 m. J0510+1800 was used as the bandpass and phase calibrators, and the quasar J0522-3627 was used for the flux calibrator.

Additionally, RW Aur was observed in Band 7 (338 GHz) on UT 2016 July 23 as part of the cycle 3 Alma proposal for a total integration time of 22.8 minutes with a $16-1110 \mathrm{~m}$ baseline range. Only 36 antennas were used in this observation. $\mathrm{J} 0510+1800$ was used as both bandpass and flux calibrator, while J0512+2927 was observed for phase calibration.

The data were initially calibrated by the NAASC. Two rounds of phase-only self-calibration were then applied to each set of observations in CASA 4.3.1 using the RW Aurigae continuum emission as the self-calibration model. The Band 6 observations were concatenated and imaged in CASA 4.3.1 using the CLEAN task. The continuum data were imaged using Briggs weighting with a robust value of 0 , yielding a synthesized beam of 0 ". $194 \times 0$ ". 077 , a beam position angle of $-12^{\circ}$, and an rms of $38 \mu \mathrm{Jy} \mathrm{bm}^{-1}$ (Figure 1). The ${ }^{12} \mathrm{CO} 2-1$ line was imaged at a velocity resolution of $0.5 \mathrm{~km} \mathrm{~s}^{-1}$, a robust value of 0.5 , and with a $u v$-taper of 0 ". $06 \times 0$ ". 25 to force a more circular beam and improve the signal-to-noise ratio $(\mathrm{S} / \mathrm{N})$, resulting in a synthesized beam of 0 ." $30 \times 0$ ". 25 , a beam position angle of $-30^{\circ}$, and an $\mathrm{rms}$ of $1.5 \mathrm{mJy} / \mathrm{bm}$ (Figure 2). The ${ }^{13} \mathrm{CO} 2-1$ line was identically imaged, resulting in a synthesized beam of 0 .! $27 \times 0$." 23 , a beam position angle of $-32^{\circ}$, and an rms of $2.0 \mathrm{mJy} /$ beam per $0.5 \mathrm{~km} \mathrm{~s}^{-1}$ channel (Figure 4).

The Band 7 continuum data were similarly imaged using Briggs weighting with a robust value of 0 , yielding a synthesized beam of 0 ." $271 \times 0$ ". 175 , a beam position angle of $-7^{\circ}$, and an rms of $560 \mu \mathrm{Jy} / \mathrm{bm}$ (Figure 1). The ${ }^{12} \mathrm{CO} 3-2$ line was imaged at a velocity resolution of $0.5 \mathrm{~km} \mathrm{~s}^{-1}$, a robust value of 0.5 , and with a $u v$-taper of 0 ." $06 \times 0$ ". 25 to match the 0 ." $30 \times 0$." 25 beam from the Band 6 observations, yielding an $\mathrm{rms}$ of $13.5 \mathrm{mJy} / \mathrm{bm}$ and a beam position angle of $-13^{\circ}$ (Figure 3). The ${ }^{13} \mathrm{CO} 3-2$ line was similarly imaged, resulting 


\section{Band 6 Continuum}

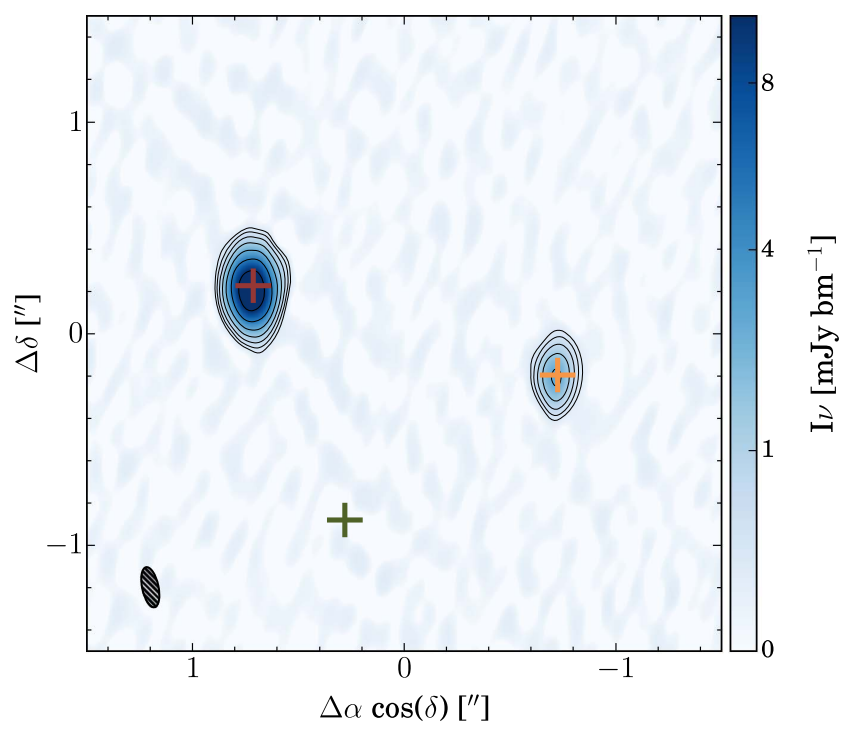

Band 7 Continuum

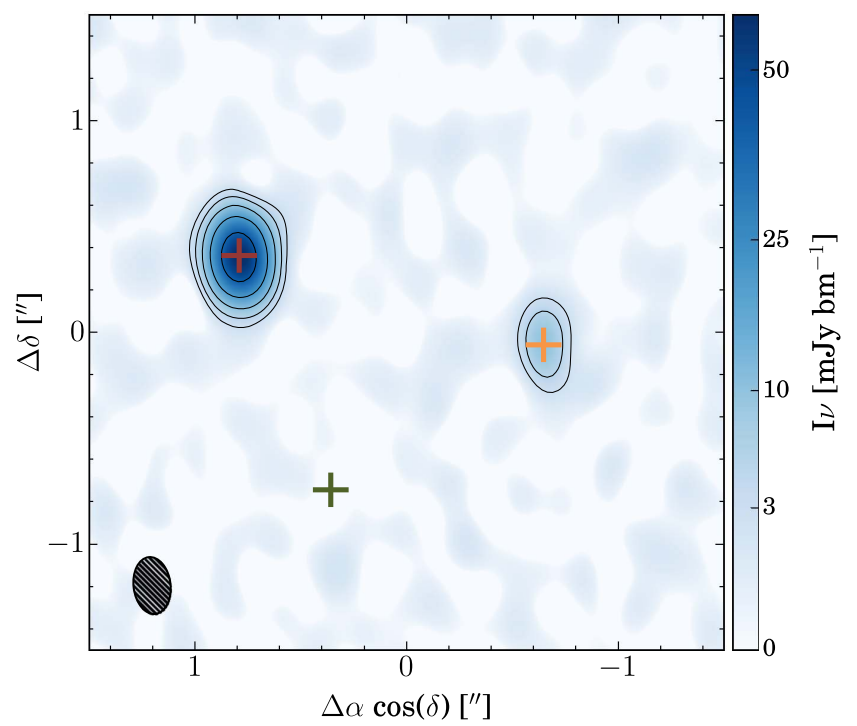

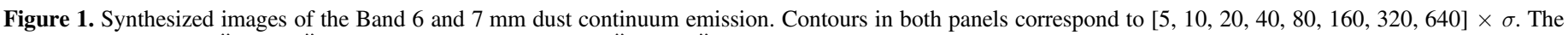

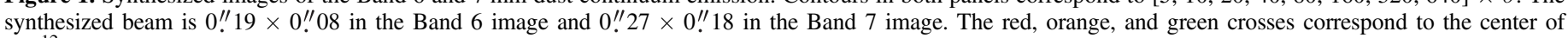
the ${ }^{12} \mathrm{CO}$ peaks for RW Aur A, B, and a third emission source, respectively.

(The data used to create this figure are available.)

in a synthesized beam of 0 ! $32 \times 0$ " 26 , a beam position angle of $-15^{\circ}$, and an $\mathrm{rms}$ of $17.5 \mathrm{mJy} /$ beam per $0.5 \mathrm{~km} \mathrm{~s}^{-1}$ channel (not shown). Our final calibrated ALMA observations are available publicly with this paper.

\subsection{Archival Observation: Kilodegree Extremely Little Telescope (KELT) and Wesleyan University}

We include and analyze the archival observations of RW Aur from the Kilodegree Extremely Little Telescope (KELT) survey and the Wesleyan University's Van Vleck Observatory that were used to first identify that RW Aurigae was experiencing long-duration, large amplitude dimming events (Rodriguez et al. 2013). The Wesleyan photometric archive ${ }^{12}$ for T-Tauri stars is described in Herbst et al. (1994). See Rodriguez et al. (2013) for a description of the KELT observations on RW Aurigae.

\subsection{ASAS-SN}

Using two fully robotic units, each consisting of four telescopes, the ASAS-SN is monitoring the entire sky down to a visual magnitude of $\sim 17$ (Shappee et al. 2014; Kochanek et al. 2017). Each telescope is a $14 \mathrm{~cm}$ Nikon telephoto lens equipped with a $2 \mathrm{k} \times 2 \mathrm{k}$ thinned $\mathrm{CCD}$, and the eight telescopes together can cover $\sim 20,000 \mathrm{deg}^{2}$ each night. The survey is designed to discover new supernovae and transient sources. Each telescope has a $8.8 \times 8^{\circ} .8$ field of view and a 7!" 8 pixel scale. ASAS-SN observed RW Aur from UT 2015 March 03 to UT 2018 March 18, obtaining 591 observations in the $V$ band. The median per point error is $0.009 \mathrm{mag}$.

\subsection{AAVSO}

Dedicated to the understanding of variable stars, the AAVSO is an amateur-professional network of $\mathrm{CCD}$ and visual

\footnotetext{
${ }^{12}$ https://wesfiles.wesleyan.edu/home/wherbst/web/TTauriDataBase/
}

observers worldwide. The AAVSO has photometric observations from UT 1906 December 18 to UT 2018 March 22, totaling 15,057 visual and $V$-band CCD observations on RW Aur. Only some of the observations have a reported uncertainty. The observations on RW Aurigae are publicly available for the community. ${ }^{13}$

\section{ALMA Results}

In this section, we describe the new cycle 3 and 4 ALMA observations of RW Aurigae that suggest we are observing the aftermath of multiple eccentric flyby interactions by RW Aur B, as first proposed by Cabrit et al. (2006).

\subsection{Continuum Modeling}

To trace the dust in the RW Aurigae system, we analyze both the Band 6 and 7 continuum observations (see Figure 1). These observations spatially resolve the disks around both RW Aur A and B; this is the first time that RW Aur B has been resolved. To best interpret this emission, we fit a simple model consisting of two Gaussian disks directly to the measured visibilities. For each disk, we fit for a position angle, inclination, integrated flux density, central offset, and a Gaussian width as a proxy for disk radius. This model used a total of 12 parameters and was fit to the observed visibilities using the Markov chain Monte Carlo (MCMC) routine emcee (Foreman-Mackey et al. 2013) combined with the visibility sampling routine vis_sample (Loomis et al. 2018). ${ }^{14}$ The best-fit model resulted in a reduced $\chi^{2}$ of 1.02, and the fit parameters are shown in Table 1.

The dust disk emission radius is compact around both sources, $\simeq 20 \mathrm{au}$, with a $30 \%$ smaller radius for source $\mathrm{B}$ at longer wavelengths. The A and B disks have similar PAs

\footnotetext{
13 https://www.aavso.org/

14 vis sample is publicly available at https://github.com/AstroChem/ vis_sample or in the Anaconda Cloud at https://anaconda.org/rloomis/vis sample.
} 


\section{CO 2-1 Moment-0}
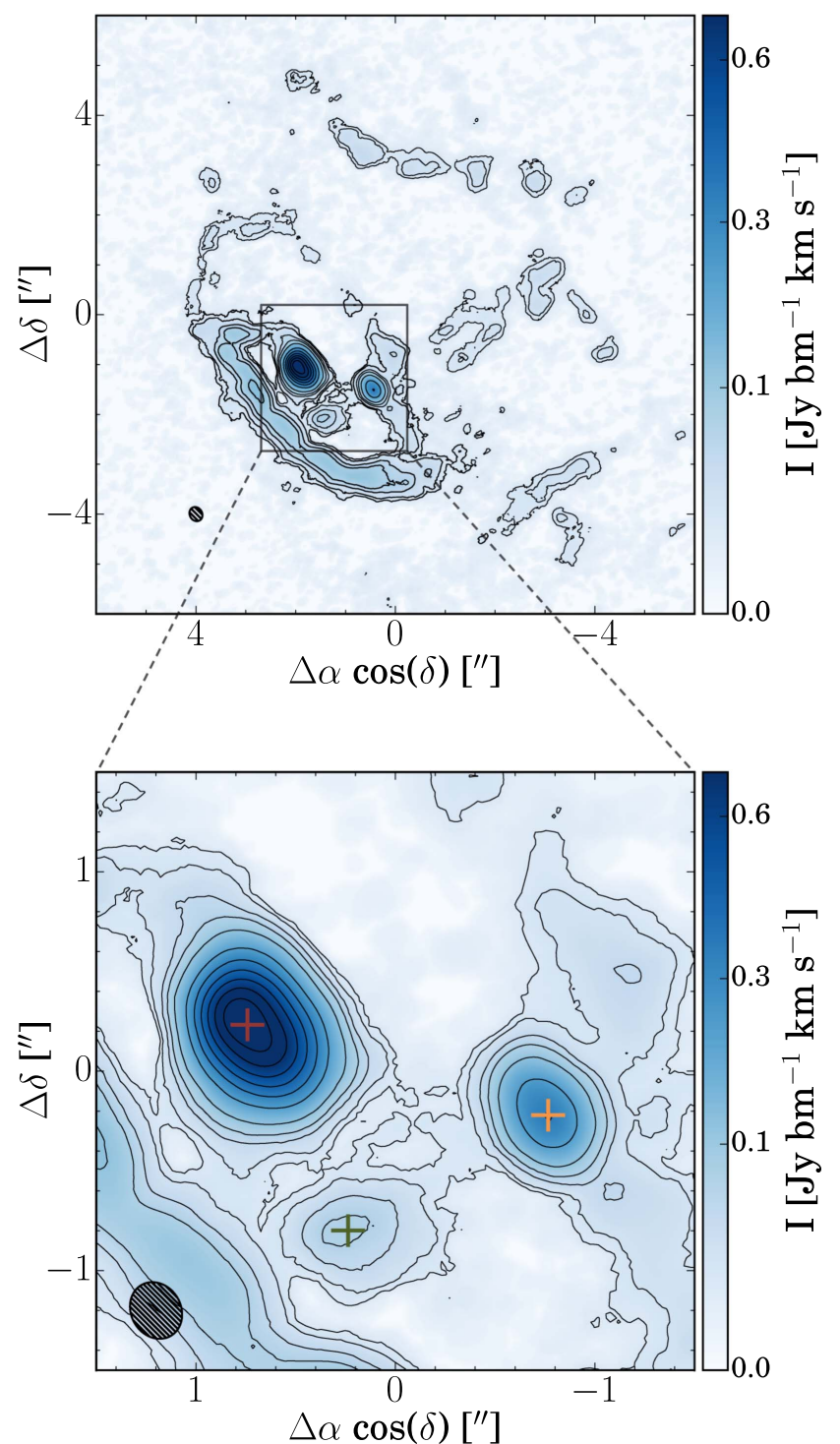

12CO 2-1 Moment-1
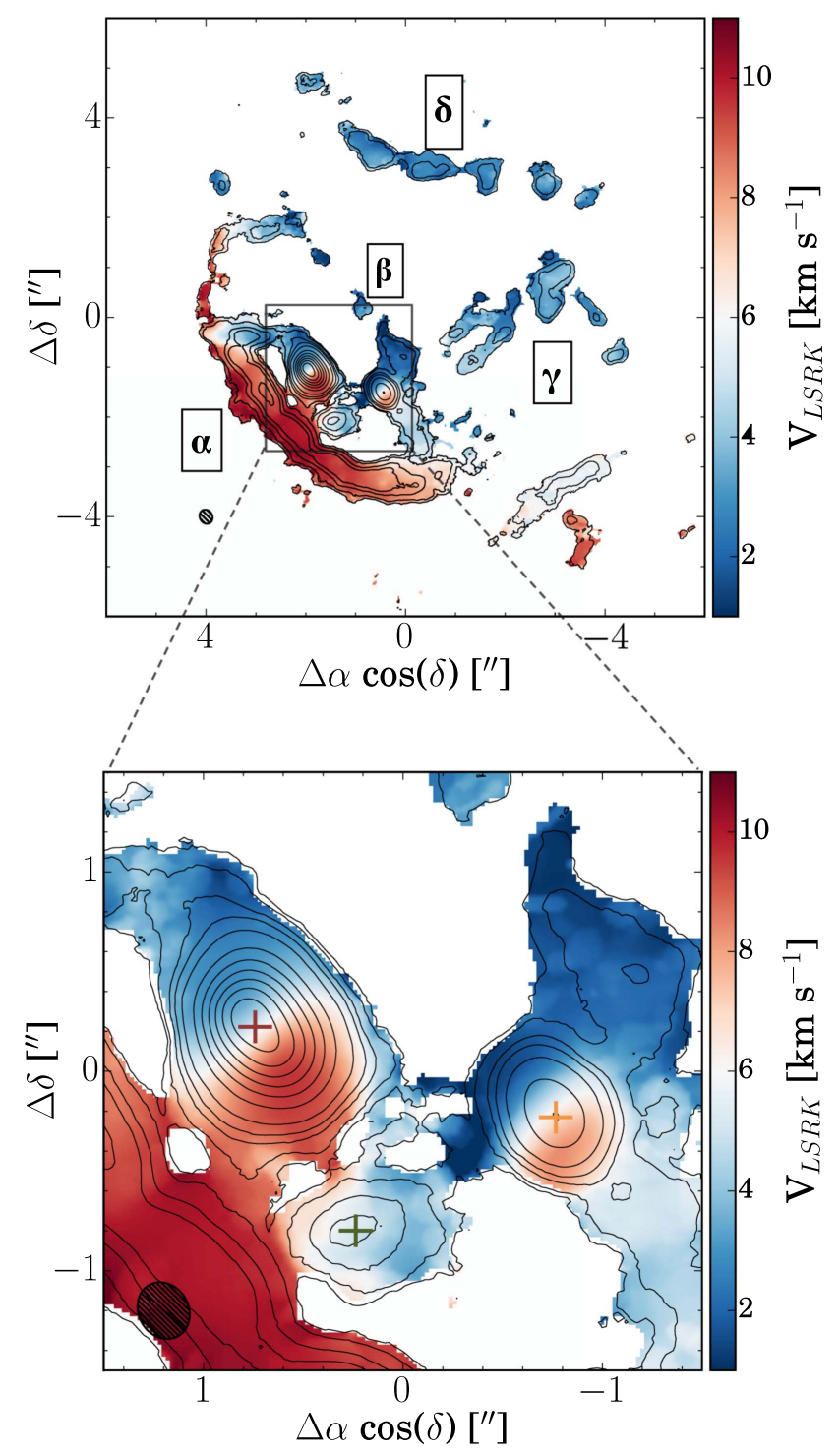

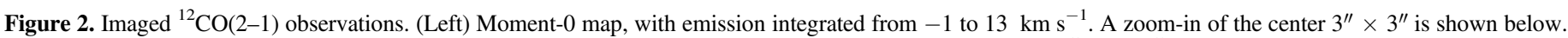

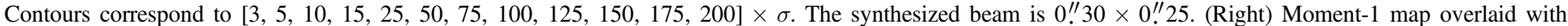

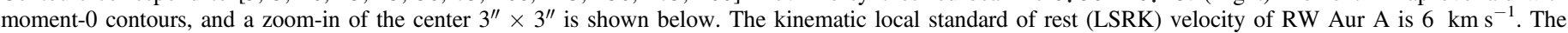

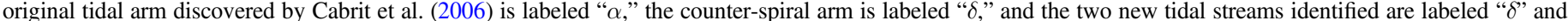

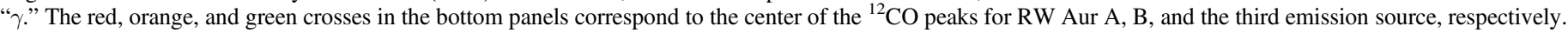
(The data used to create this figure are available.)

(within 1 sigma), with the disk around RW Aur A perpendicular to its optical jet $\left(\mathrm{PA}=130^{\circ} \pm 2^{\circ}\right.$, Dougados et al. 2000). In contrast, the disk inclinations differ by $12^{\circ}$ (6 sigma) in Band 6 (and $15^{\circ} \pm 8^{\circ}$ in Band 7). It seems difficult to attribute this difference to residual random phase noise, as we would expect this effect to make the smaller disk (B) appear rounder and less inclined than the larger one (A). We therefore conclude that the disks around RW Aur A and B appear to be misaligned relative to each other by roughly $12^{\circ}$ if their upper surfaces both face to the SE (the direction of the blueshifted jet of RW Aur A), or by $57^{\circ}$ if they face in opposite directions. Other young binary systems where both stars have a circumstellar disk have also been shown to be misaligned relative to each other (e.g., Jensen \& Akeson 2014, and references therein).
Our disk fits give a separation between RW Aur A and B of 1 ". $497 \pm 0$ ". 001 in Band 6 and 1".490 \pm 0 ".010 in Band 7, which is in agreement with the $\mathrm{AB}$ separation measured by Cabrit et al. (2006) of 1". $468 \pm 0$ !" 056 , but larger than the optical Hubble Space Telescope (HST) separation of 1". $4175 \pm 0$ ". 0034 (Ghez et al. 1997). The separation of RW Aur A and B was also measured with Chandra to be 1" $48 \pm 0$ ".01 (Skinner \& Güdel 2014). Recently, Gaia measured the separation to be $1.4863 \pm 0$ ".0006 (Gaia Collaboration et al. 2016). Using a large compilation of data from the literature, Csépány et al. (2017) determined that RW Aur A and B are currently moving away from each other at

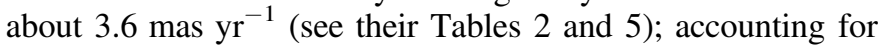
this increase in separation places our observations in excellent agreement with the HST data of Ghez et al. (1997), and with the 


\section{CO 3-2 Moment-0}

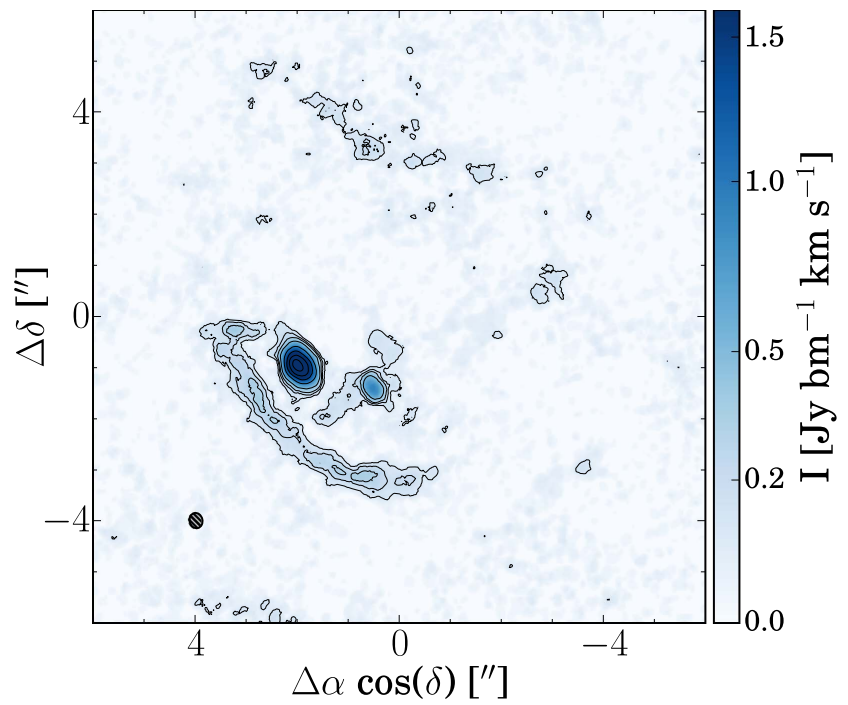

12CO 3-2 Moment-1

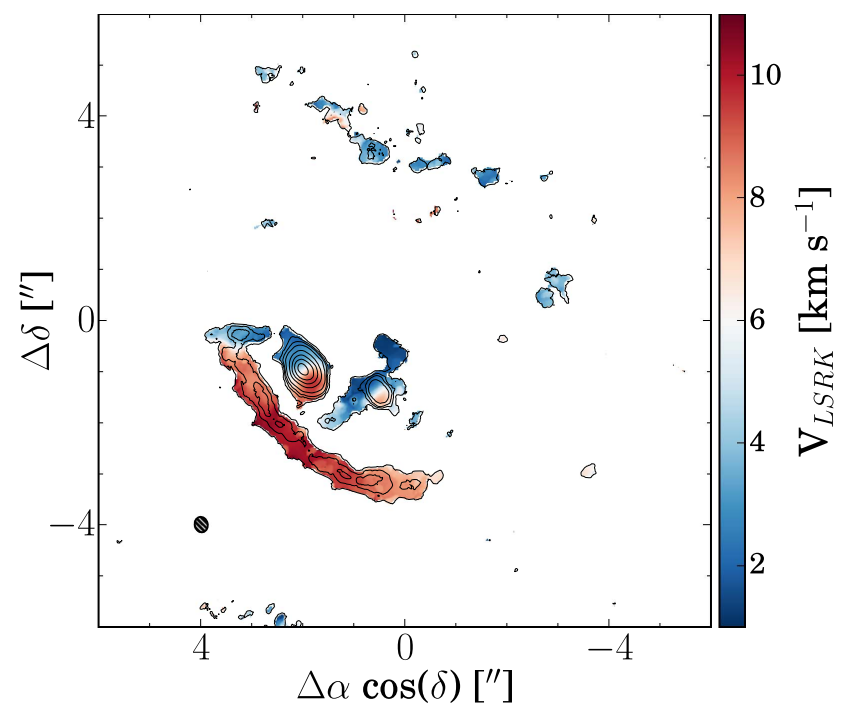

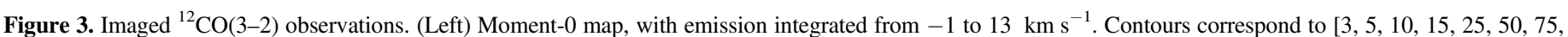

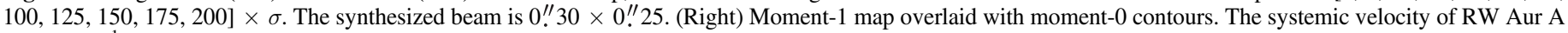
is $6 \mathrm{~km} \mathrm{~s}^{-1}$.

(The data used to create this figure are available.)

other accurate separation measurements obtained in the meantime with the Nasmyth Adaptive Optics System (NAOS) NearInfrared Imager and Spectrograph (CONICA; together known as $\mathrm{NaCo}$ ) at the Very Large Telescope (VLT/NaCo; Correia et al. 2006), CHANDRA (Skinner \& Güdel 2014), and Gaia (Gaia Collaboration et al. 2016).

Our total Band 6 flux density for the RW Aur A and B system is $40.92 \pm 0.03 \mathrm{mJy}(\mathrm{RW}$ Aur $\mathrm{A}=36.31 \pm 0.02 \mathrm{mJy}$ and RW Aur B $=4.61 \pm 0.02 \mathrm{mJy}$ ), which is consistent with the $1.3 \mathrm{~mm}$ total system flux density of $42 \pm 2 \mathrm{mJy}$ measured by Osterloh et al. (1996), but is $25 \%$ higher than the $32.8 \pm 0.7 \mathrm{mJy}(\mathrm{RW}$ Aur $\mathrm{A}=27.6 \pm 0.5 \mathrm{mJy}$ and RW Aur $\mathrm{B}=5.2 \pm 0.4 \mathrm{mJy}$ ) determined by Cabrit et al. (2006). This discrepancy is compatible with the absolute flux calibration accuracy of $\simeq 20 \%$ estimated in Cabrit et al. (2006) for their PdBI observations. Their lower flux ratio of $\mathrm{A} / \mathrm{B}$ at $1.3 \mathrm{~mm}$ (5, instead of 8 in the ALMA data) could result from the fact that RW Aur B was detected at a low $\mathrm{S} / \mathrm{N}$ on top of a faint emission bridge connecting to RW Aur A, which may have caused an overestimate of the B flux density (this bridge is not seen in the more sensitive ALMA data, and was thus likely a cleaning artifact). In Band 7, our total measured flux density of $97.8 \pm 0.5 \mathrm{mJy}$ is $20 \%-30 \%$ higher than the total system flux density at 863 microns of $70 \pm 4$ mJy measured with SCUBA in single-dish images by Andrews \& Williams (2005), again consistent within the absolute flux calibration errors.

\subsubsection{Spectral Index}

Comparing the ALMA Band $6(225 \mathrm{GHz})$ and Band 7 (338 GHz) flux densities, we find a spectral index $\left(\alpha=\Delta \log F_{\nu} / \Delta \log \nu\right)$ of $2.11 \pm 0.01$ in RW Aur $\mathrm{A}$ and $2.39 \pm 0.07$ in RW Aur B. In both sources, the PdBI 122.5 GHz continuum flux densities from Cabrit et al. (2006) are consistent with an extrapolation along these slopes, indicating a constant spectral index across this wavelength range for both disks. The shallower spectral index of $1.73 \pm 0.08$ reported for RW Aur A by Cabrit et al. (2006) was probably caused by their $25 \%$ underestimate in absolute flux calibration at $1.3 \mathrm{~mm}$ (see discussion in previous paragraph). We note that for a disk of radius 20 au viewed at a 55 degrees of inclination, the mean brightness temperature required to reproduce the observed continuum flux in RW Aur $\mathrm{A}$ in Band 7 is about $39 \mathrm{~K}$; this value matches the dust temperature at 20 au very well that was predicted by fits to the infrared spectral energy distribution (SED) of RW Aur A, $T_{d}(r)=217 \mathrm{~K} \quad(r / \mathrm{au})^{-0.57} \quad$ (Osterloh \& Beckwith 1995). Hence, the dust emission in RW Aur A may be optically thick at $338 \mathrm{GHz}$. This would be consistent with the spectral slope of $1.43 \pm 0.21$ reported by Andrews \& Williams (2005) for the total system flux density (dominated by A) between 850 and $380 \mu \mathrm{m}$ (a blackbody at $39 \mathrm{~K}$ predicts an index of 1.6 in this frequency range). At longer wavelengths, the observed spectral index $\alpha \simeq 2.11$ between ALMA Band 7 and Band 6 is slightly steeper than the value of 1.8 predicted for a $39 \mathrm{~K}$ blackbody, indicating that the emission is becoming optically thin below $200 \mathrm{GHz} .{ }^{15}$ In RW Aur B, the flux density for a similar dust emission radius $\simeq 20$ au is eight times weaker, which indicates that dust continuum is optically thin even in Band 7, which in turn is consistent with the steeper slope of 2.4 .

\subsection{Spectral Line Emission}

To map out the kinematics and size distributions of the gas in the RW Aurigae system, we analyze the ${ }^{12} \mathrm{CO}(2-1,3-2)$, ${ }^{13} \mathrm{CO}(2-1,3-2)$ (see Figures $\left.2-4\right), \mathrm{C}^{18} \mathrm{O}(2-1)$, and $\mathrm{SO}(2-1)$ emission (not shown). Only a small amount of $\mathrm{C}^{18} \mathrm{O}(2-1)$ emission is detected toward RW Aur A, and none is detected toward RW Aur B. We do not detect any $\mathrm{SO}(2-1)$ emission from either source. Our analysis of the ${ }^{12} \mathrm{CO}(2-1)$ emission (see the Moment-1 map in Figure 2) reveals numerous features: disks around RW Aur A and RW Aur B, the original tidal arm (labeled " $\alpha$ " in the Moment-1 map), and a second blueshifted

\footnotetext{
15 For example, if we crudely approximate the disk as a graybody at $39 \mathrm{~K}$, the observed flux densities could be reproduced with an optical depth $\tau_{\nu} \simeq 3$ $(\nu / 338 \mathrm{GHz})^{0.8}$.
} 
Table 1

Results of Continuum and Spectral Line Fitting

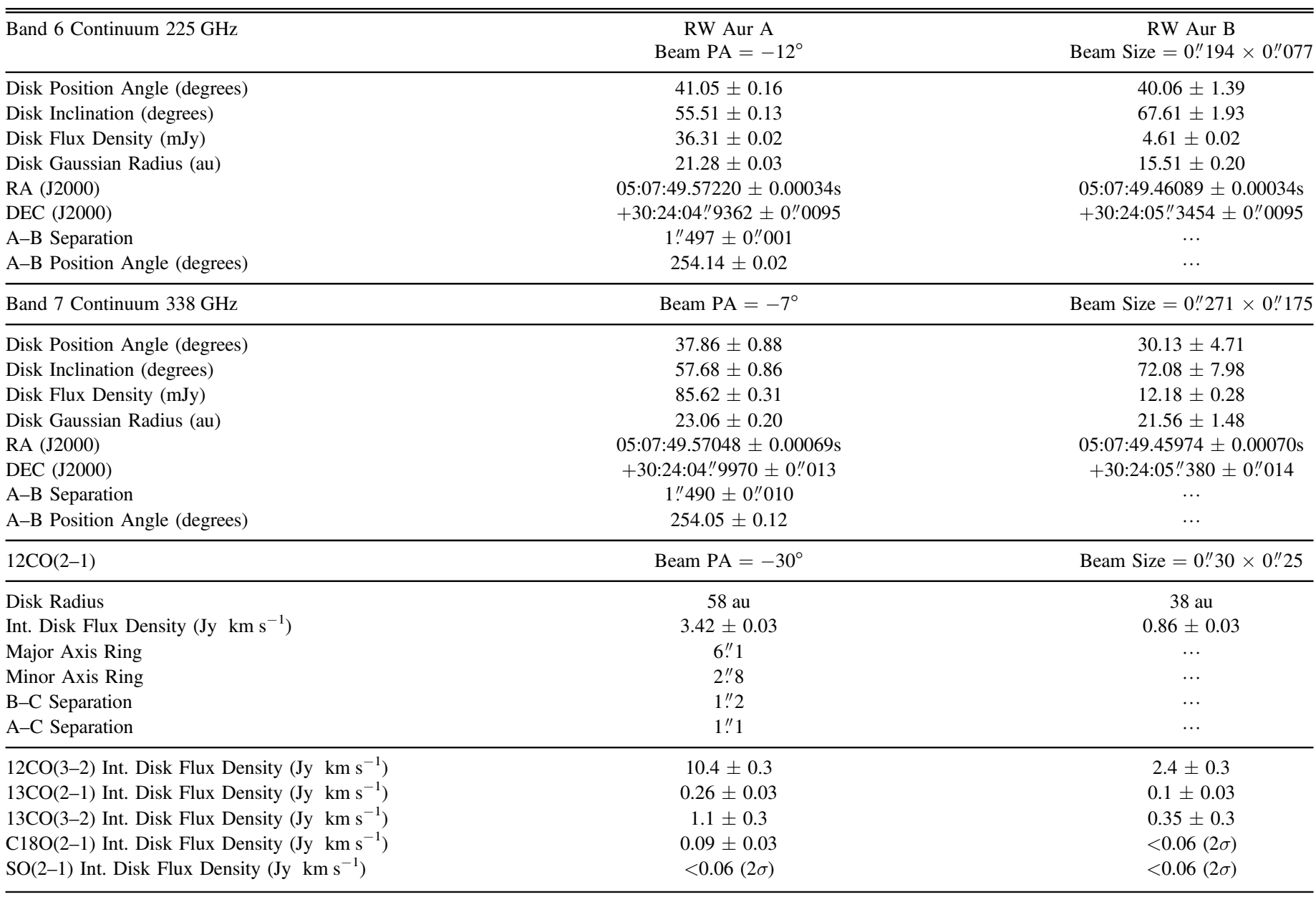

Note. Measurements on the continuum were made by fitting two elliptical Gaussian disks in the $(u, v)$ plane (see Section 2). Source C was not detected in the continuum. Errors on the gas flux densities are likely underestimated as distinguishing which feature contributes flux is not trivial.

counter-arm forming an apparent ring of emission with the main one (labeled " $\beta$ " in the Moment-1 map), a second tidal stream that is inclined relative to the apparent circumbinary ring (labeled " $\delta$ " in the Moment-1 map), a third tidal stream (labeled " $\gamma$ " in the Moment-1 map), and a third clump of emission originating between RW Aur A, B, and the original tidal arm. Below we discuss the possible origin for this clump of emission.

\subsubsection{RW Aur A}

RW Aur A is detected in ${ }^{12} \mathrm{CO}\left(2-1=3.42 \pm 0.03 \mathrm{Jy} \mathrm{km} \mathrm{s}{ }^{-1}\right.$, $\left.3-2=10.4 \pm 0.3 \mathrm{Jy} \mathrm{km} \mathrm{s}^{-1}\right),{ }^{13} \mathrm{CO}\left(2-1=0.26 \pm 0.03 \mathrm{Jy} \mathrm{km} \mathrm{s}^{-1}\right.$, $\left.3-2=1.1 \pm 0.3 \mathrm{Jy} \quad \mathrm{km} \mathrm{s}^{-1}\right), \quad$ and $\quad \mathrm{C}^{18} \mathrm{O}(2-1) \quad(0.09 \pm 0.03$ Jy $\mathrm{km} \mathrm{s}^{-1}$ ). We place a $2 \sigma$ upper limit on SO (2-1) emission of $<0.06 \mathrm{Jy} \mathrm{km} \mathrm{s}^{-1}$.

A clear Keplerian velocity gradient is observed across the disk around RW Aur A, consistent with a stellar mass range of 1-1.5 $M_{\odot}$, assuming a PA of $41^{\circ}$ and a dust disk inclination of $55^{\circ}$ from our $(u, v)$ fit of the Band 6 continuum (see Figure 6 ). This mass range is consistent with the inferred mass of 1.3-1.4 $M_{\odot}$ determined from pre-main sequence models of near-IR observations (Ghez et al. 1997; Woitas et al. 2001). Using the CASA built-in measurement tool (McMullin et al. 2007), we estimate the radius of the gas disk around RW Aur A from the ${ }^{12} \mathrm{CO}(2-1)$ to be $\sim 58$ au, $\sim 2.5$ times larger than the extent of the $\mathrm{mm}$ continuum emission.

${ }^{12} \mathrm{CO}(2-1)$ and (3-2) spectra (see Figure 5) were extracted from the image cubes using an elliptical mask with a size equivalent to the measured CO extent of RW Aur A shown in Table 1. The line profiles are double peaked, as expected for a Keplerian disk, but are not symmetric about the LSRK systemic velocity (estimated as $V_{\mathrm{LSRK}}(A) \simeq 16 \mathrm{~km} \mathrm{~s}^{-1}$ ). The blueshifted side of the peak is broader and shallower than the redshifted side relative to the motion of the star. This observed asymmetry in the integrated spectra may be caused by material being sheared off the disk into the tidal arm, as seen in the Moment- 1 maps. Based on our results, the ratio of ${ }^{12} \mathrm{CO}(3-2) /(2-1) \sim 3.0$.

The peak surface brightness in ${ }^{12} \mathrm{CO}(2-1)$ of $S_{\nu}=$ $190 \mathrm{mJy} /$ beam translates into a peak brightness temperature $T_{b}=58 \mathrm{~K}$. This indicates that the CO-emitting gas at $60 \mathrm{au}$ in the RW Aur A disk is significantly warmer than the dust at $20 \mathrm{au}$ (for which continuum flux densities were found consistent with a temperature $\sim 40 \mathrm{~K}$ ). This result confirms the conclusion of Cabrit et al. (2006) that CO emission in the RW Aur A disk appears to probe a warm super-heated disk surface layer located above the cool midplane probed by millimeter dust emission. ALMA studies have clearly resolved the vertical increase in temperature in the atmosphere of disks on scales of 


\section{CO 2-1 Moment-0}

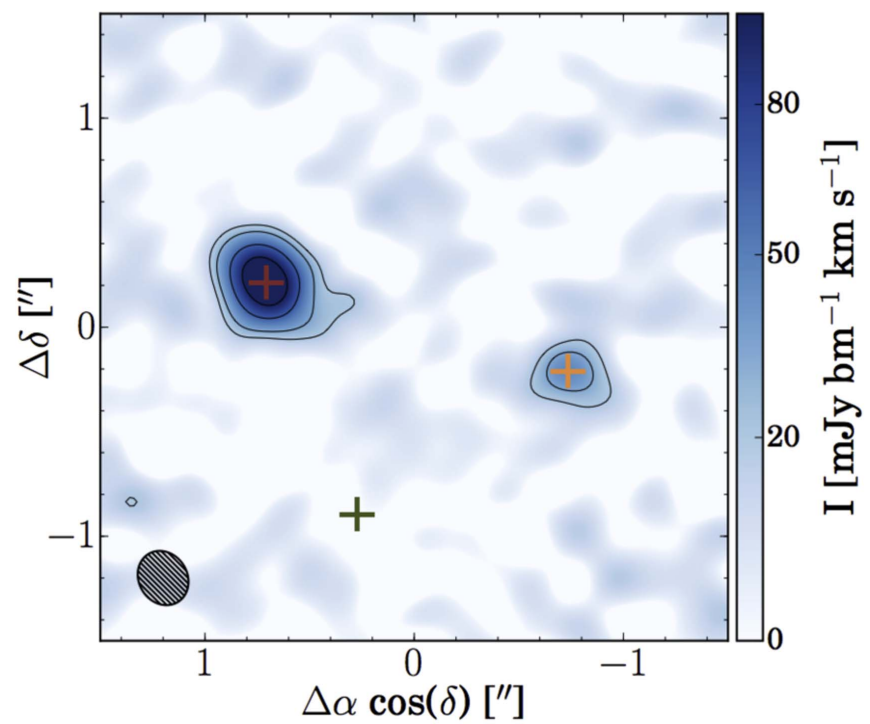

Figure 4. Moment- 0 map of ${ }^{13} \mathrm{CO}(2-1)$ emission, integrated from -1 to 13 $\mathrm{km} \mathrm{s}^{-1}$. Contours correspond to $[3,5,10,15] \times \sigma$. The synthesized beam is 0 ! $27 \times 0$ ! 23 . The red, orange, and green crosses correspond to the center of the ${ }^{12} \mathrm{CO}$ peaks for RW Aur A, B, and the third emission source, respectively. (The data used to create this figure are available.)

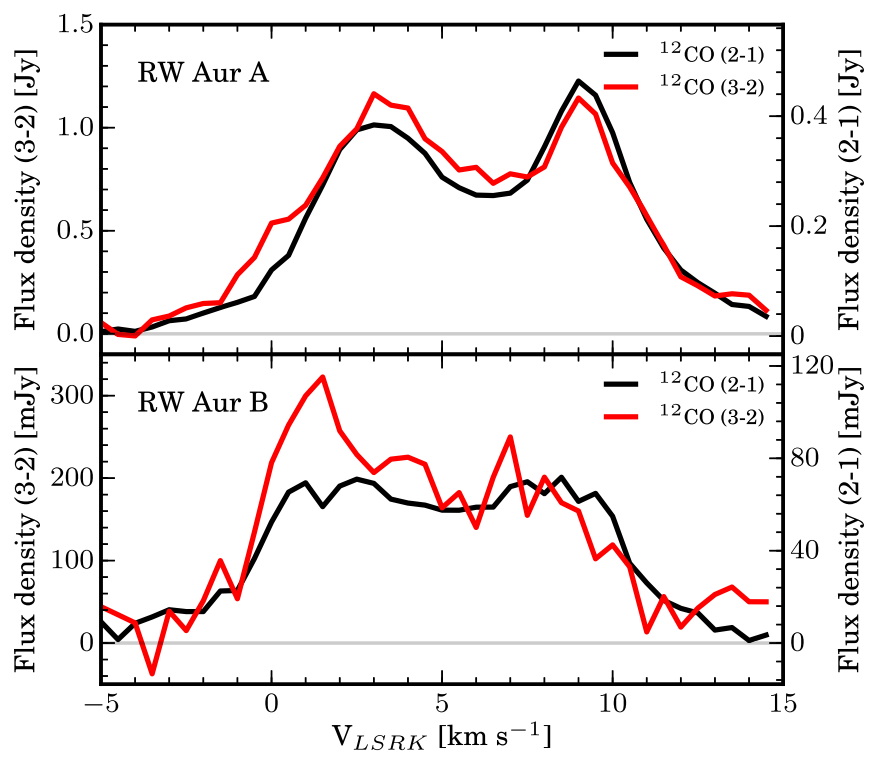

Figure 5. ${ }^{12} \mathrm{CO}$ spectra of RW Aur A (top) and B (bottom). The $J=2-1$ spectrum is shown in black and the $J=3-2$ spectrum is shown in red. The $J=2-1$ spectra for RW Aur A and B have been multiplied by the $(3-2) /(2-1)$ ratios in Table 1. An elliptical mask with a size equivalent to the measured $\mathrm{CO}$ extent was used to extract the spectra.

100-200 au (Pinte et al. 2018), but the tidal truncation of the disk in RW Aur allows us to probe this effect at much smaller radii.

\subsection{2. $R W$ Aur B}

RW Aur B was only detected in ${ }^{12} \mathrm{CO}(2-1=0.86 \pm 0.03$ Jy $\left.\mathrm{km} \mathrm{s}^{-1}, 3-2=2.4 \pm 0.3 \mathrm{Jy} \mathrm{km} \mathrm{s}^{-1}\right)$ and ${ }^{13} \mathrm{CO}(2-1=$ $0.1 \pm 0.03 \mathrm{Jy} \mathrm{km} \mathrm{s}^{-1}, 3-2=0.35 \pm 0.3 \mathrm{Jy} \mathrm{km} \mathrm{s}^{-1}$ ). The PV diagram for RW Aur B suggests Keplerian rotation corresponding to a stellar mass of $0.5 M_{\odot}<M<1 M_{\odot}$, assuming an inclination of $79^{\circ}$ and a PA of $46^{\circ} .4$ based on our continuum fitting (see Figure 6). This mass range is consistent with the inferred mass of $0.7-0.9 M_{\odot}($ Ghez et al. 1997; Woitas et al. 2001). We estimate the radius of the gas disk around RW Aur B in ${ }^{12} \mathrm{CO}(2-1)$ to be 38 au, $~ 2.5$ times larger than its dust disk.

Although the integrated line profile is noisier for RW Aur B (see Figure 5), we do detect a double-peaked Keplerian profile for both ${ }^{12} \mathrm{CO} J=3-2$ and $J=2-1$ around a systemic velocity of about $5.2 \mathrm{~km} \mathrm{~s}^{-1}$, blueshifted by $-0.8 \mathrm{~km} \mathrm{~s}^{-1}$ from $\mathrm{A}$. The observed ${ }^{12} \mathrm{CO}(3-2) /(2-1)$ ratio is 2.8 .

The peak surface brightness in ${ }^{12} \mathrm{CO}(2-1)$ of $\sim 50 \mathrm{mJy} /$ beam translates into a brightness temperature $T_{b}=15 \mathrm{~K}$. The disk of RW Aur B is only marginally resolved by our beam (radius of 38 au $\simeq 0$ "' 27). Therefore, the observed peak surface brightness probably suffers substantial beam dilution, and the true brightness is higher than this.

\subsubsection{Tidal Streams}

Observations from IRAM PdBI discovered a long tidal stream wrapped around RW Aur A, suggesting that RW Aur had previously undergone an eccentric star-disk encounter (Cabrit et al. 2006). These observations resembled the properties of simulated stellar fly-bys on an accretion disk (Clarke \& Pringle 1993). Therefore, it was proposed that RW Aur B is on a prograde, highly eccentric orbit causing it to approach RW Aur A very closely, possibly within the extent of the original circumstellar disk around RW Aur A. It was determined that unlike previously observed circumbinary rings, the velocity structure of the arm was inconsistent with pure rotation. The $\mathrm{NE}$ and SW extension of the arm is blueshifted, while the middle of the arm is redshifted. Cabrit et al. (2006) suggested that the arm is likely expanding outward from RW Aur.

The new ${ }^{12} \mathrm{CO}$ emission maps (see Figures 2 and 3) clearly show the original tidal arm (labeled " $\alpha$ " in the Moment-1 map shown in Figure 2) found by Cabrit et al. (2006) and confirm that the NE extension is blueshifted, while most of the arm is redshifted with respect to the systemic velocity. The ALMA observations resolve the arm, showing evidence of possible clumpy substructure along its length. Given the recent occultation events, which may suggest that material is coalescing, it is not surprising to see substructure within the disrupted gas shown in the ${ }^{12} \mathrm{CO}$ maps. The arm is connected to the NE portion of the RW Aur A disk and is not detected in the continuum. Based on the ${ }^{12} \mathrm{CO}(2-1)$ map, we estimate the arm to be $\sim 740$ au along the spine of the arm $(\sim 5$ !' 2$)$, having an estimated typical width of $\sim 85$ au $(\sim 0$." 6$)$. We note that the arm fluctuates in its apparent width by $\sim 0$ !" 1 and has a total integrated flux density in the ${ }^{12} \mathrm{CO}(2-1)$ of $3661 \pm$ $58 \mathrm{mJy} \mathrm{km} \mathrm{s}^{-1}$. It appears that this tidal stream wraps fully around RW Aur A and B, forming the appearance of a circumbinary ring of gas. We note that this is likely a projection effect and that there is actually a second counter-spiral around RW Aur B from its flyby (labeled " $\beta$ "). In Section 5 we discuss the nature and morphology of these tidal streams, providing evidence to favor the dual spiral arm interpretation.

In addition to the main tidal arm $(\alpha)$ first identified by Cabrit et al. (2006) and the secondary arm $\beta$, we identify two additional tidal streams extending out past the extent of the apparent circumbinary ring (labeled " $\gamma$ " and " $\delta$ " in the 

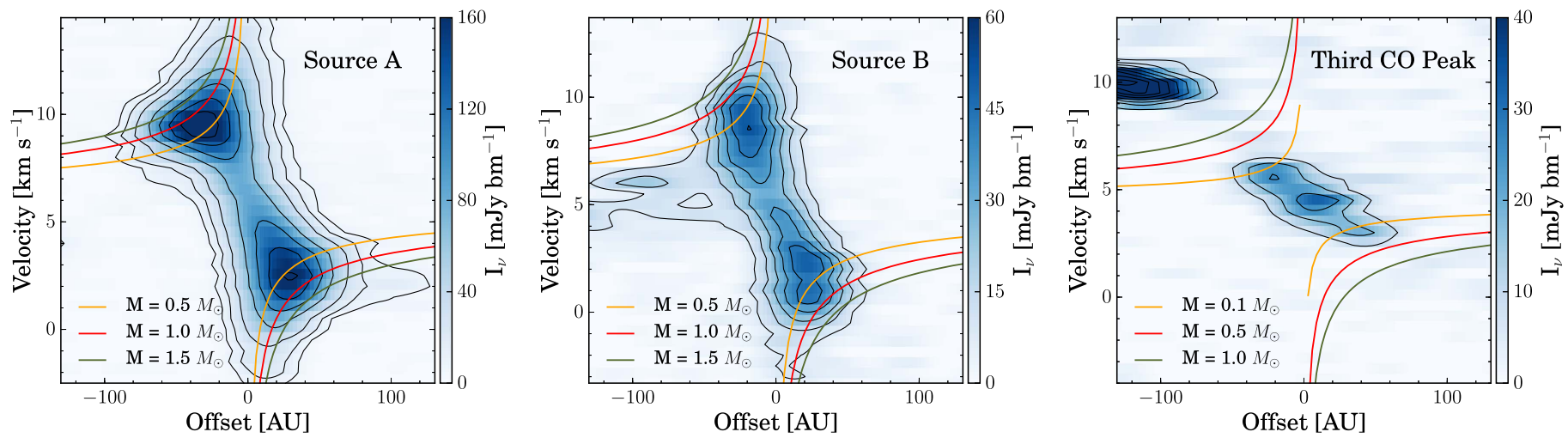

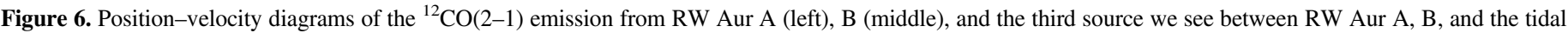

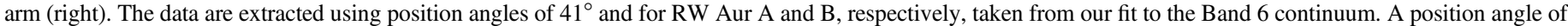

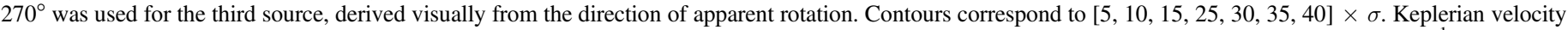

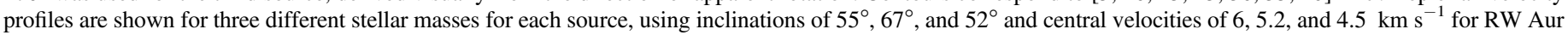
$\mathrm{A}, \mathrm{B}$, and the third source, respectively.

Moment-1 map shown in Figure 2). For clarity, we refer to these two features as "tidal streams" in comparison to the two features that form the projected circumbinary ring, which we refer to as "spirals" or "arms." It is possible that these two new tidal streams are actually one continuous stream, but our observations are not sensitive enough to constrain this due to a lack of $(u, v)$ coverage at intermediate baselines. In Section 5 we discuss whether these additional tidal streams are bound to the system.

One of the new tidal streams ( $\gamma$ in Figure 2) appears to be inclined relative to the tidal arm (possibly perpendicular). While this could be partly due to projection effects (foreshortening), we note that the orientation of the $\gamma$ stream on the sky is within a few degrees of perpendicular to the PA of the disk around RW Aur B. This coincidence raises the alternative possibility that $\gamma$ might trace a slow molecular outflow from RW Aur B. RW Aur B is also an active accretor (Duchêne et al. 1999) and could have experienced an enhanced ejection phase near periastron passage. Additional $(u, v)$ coverage at intermediate scales would be needed to test this scenario by recovering the full structure of this stream.

Finally, to the south of the two stars, we also note a bright clump of CO emission that we refer to as the "third CO peak" (see Figure 2). The linear slope of the PV diagram for this emission clump (see Figure 6) is not consistent with resolved Keplerian rotation. On the other hand, it is reminiscent of the linear PV diagram across the (unresolved) B disk in the PdBI observations of Cabrit et al. (2006). Moreover, a few PV diagrams with similar linear slopes are observed toward edgeon protostars, where they appear to trace a rotating ring near the disk centrifugal barrier (see, e.g., Figure 5 in Lee et al. 2017). The Keplerian rotation curves shown in Figure 6 would then set an upper limit of $\sim 0.1 M_{\odot}$ to the mass of a possible embedded source in the third $\mathrm{CO}$ peak.

To constrain the presence of continuum emission associated with the third ${ }^{12} \mathrm{CO}$ peak, the Band 6 observations were reimaged with natural weighting. No continuum emission was detected at this location, with a $2 \sigma$ upper limit of $30 \mu \mathrm{Jy}$. Comparing this limit with the measured ${ }^{12} \mathrm{CO} 2-1$ integrated flux density (220 mJy km s${ }^{-1}$ ), we find a $\mathrm{CO}$ to continuum ratio of $>7000 \mathrm{~km} \mathrm{~s}^{-1}$, while RW Aur A/B have ratios of 94 and $187 \mathrm{~km} \mathrm{~s}^{-1}$, respectively.
We further note that the location of this emission would likely be very unstable dynamically, unless the source was significantly in front or behind RW Aur. Therefore, if the third $\mathrm{CO}$ peak is tracing a third stellar or substellar companion in the RW Aur system, it would have an unusually low amount of millimeter-sized dust and would be on an unstable orbit. Another possible explanation would be that the third $\mathrm{CO}$ peak is connected to the $\gamma$ stream by a fainter unseen structure. Complementary $(u, v)$ coverage at intermediate baselines would be necessary to confirm or exclude this possibility.

\section{Photometric Results}

Over the past decade, the RW Aurigae system has undergone four separate dimming events that have varied in duration and depth (Rodriguez et al. 2013; Antipin et al. 2015; Petrov et al. 2015; Bozhinova et al. 2016; Rodriguez et al. 2016; Berdnikov et al. 2017). We report here a new dimming event that is currently ongoing, and we also analyze an event dating back to 1937 that was first identified by Berdnikov et al. (2017; see Figures 7 and 8). The cause of these dimmings is debated but may be due to a dusty disk wind very close to the star (Petrov et al. 2015; Shenavrin et al. 2015), disrupted circumstellar material from the eccentric flyby farther out (Rodriguez et al. 2013, 2016), variation in the geometry of the inner disk (Schneider et al. 2015; Facchini et al. 2016) similar to what has been proposed to explain the large dimmings of AA Tau (Bouvier et al. 2013; Loomis et al. 2017), or tidal perturbations of the RW Aur A disk propagating inward since the flyby and now reaching the inner 1 au (Berdnikov et al. 2017).

In this section, we summarize the eclipse parameters for each of the seven dimmings, including one that occurred in the late 1930s. We model each event separately as an occultation of RW Aur A by large opaque screen with a leading edge perpendicular to its direction of motion (following Section 5.1 in Rodriguez et al. 2013). From this analysis, we estimate the transverse velocity and width of the occulting body required to explain each dimming event separately and summarize the results in Table 2. The analysis in this section provides an estimated range of the size and velocity of the occulting dust screens to explain each event. In each dimming, we see ineclipse photometric structure that we attribute to changes in the opacity and/or vertical size of the occulting bodies. Therefore, our estimated velocities are likely a lower limit since we are 


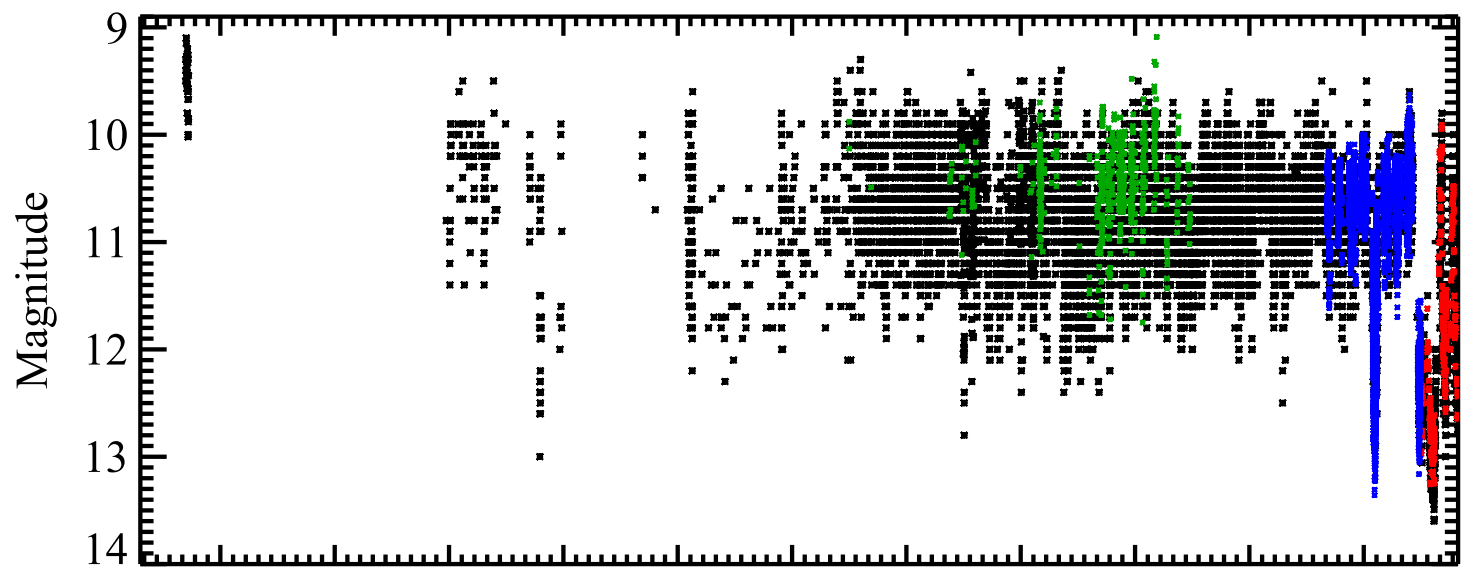

19101920193019401950196019701980199020002010

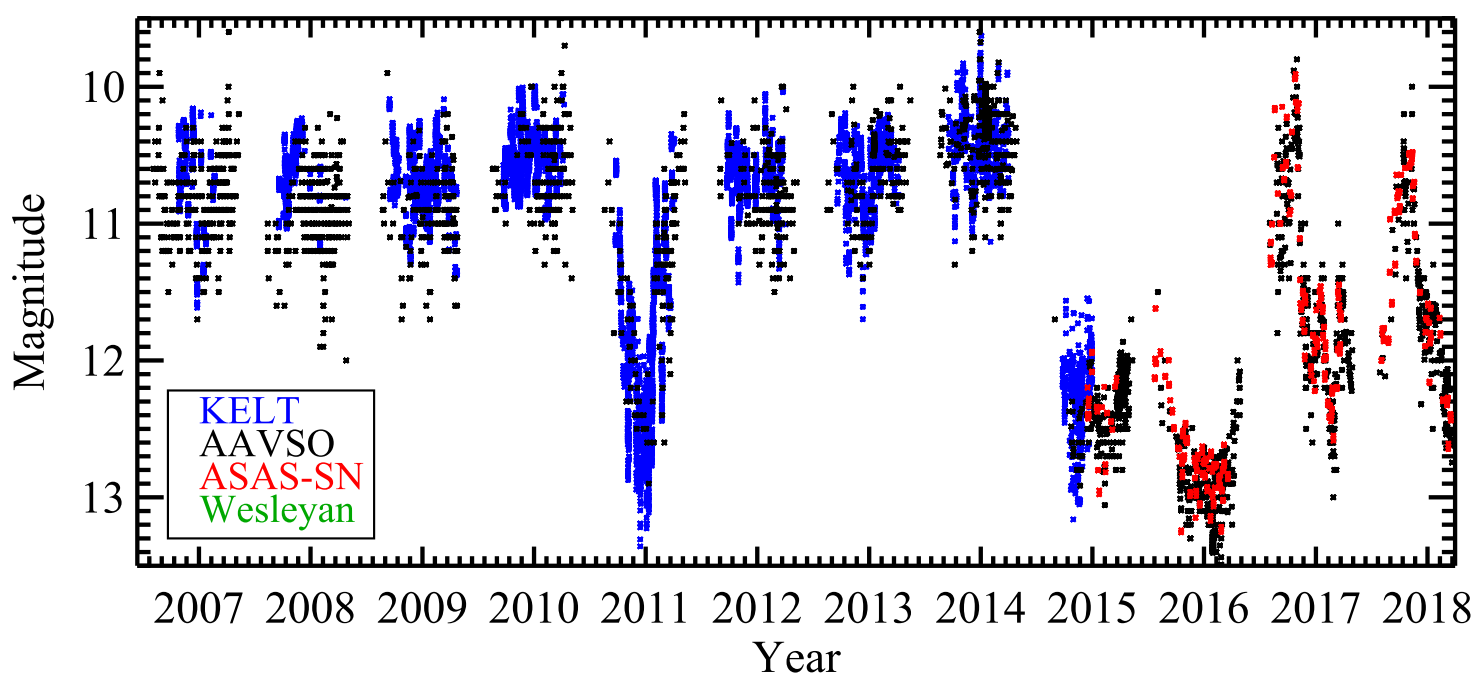

Figure 7. Recreation of Figure 1 from Rodriguez et al. (2013, 2016; top) showing the full 110-year-long lightcurve from AAVSO (black), Wesleyan (green), KELT (blue), and ASAS-SN (red) of RW Aur and (bottom) a zoom-in showing the photometric variability of the system over the last decade.

assuming a sharp leading edge of an opaque occulting screen when the observed structure during the eclipse would suggest otherwise. The lower limit on the velocity results in an upper limit on the estimated semimajor axis. The quoted capitalized letters in the following sub-section headers correspond to the same letter label shown in the bottom left corner of each panel in Figure 8. In Section 5.1, we summarize our results and discuss them in context with our analysis of the ALMA observations.

\subsection{Historical Dimming: 1937-1938 “A," 1987 “B”}

Prior to the 2010-2011 dimming, no similar event has been observed in the previous $\sim 60$ years. Although the baseline brightness of RW Aur has varied over the past 110 years (Berdnikov et al. 2017), no gap in the AAVSO observation is large enough for a 180-day event of similar depth to have been missed since 1960 (see Figure 7, Rodriguez et al. 2013) and no similar (depth and duration) event has been observed over the last $\sim 110$ years (e.g., Berdnikov et al. 2017, and references therein). However, two shallower and shorter episodes of dimmings were observed in 1937 and 1987. In early 1937 December, RW Aur dimmed by $\sim 2$ mag for $\sim 30$ days, ending in early 1938 January. During this dimming, RW Aur appeared to brighten by 1 magnitude for $\sim 10$ days during the middle of the event (see Figure 8). The initial dimming of this event was not observed in our $V$-band observations, but RW Aur takes $\sim 6$ days to recover to its normal brightness. However, the initial dimming was observed in the $B$ band, showing it to be similar in duration. Prior to the 1937-1938 event, a very short dimming event with a similar depth was seen in the $B$-band observation (Berdnikov et al. 2017). Additionally, shortduration photometric variability is also seen after this event, consistent with trailing circumstellar material to the main occultating screen. This dimming occurred during a known 10-15-year-long change in the nominal brightness of the RW Aur system (Berdnikov et al. 2017), but we note that the depth, shape, and short duration of the event argues that this is separate from the known baseline brightness change. Additionally, Berdnikov et al. (2017) identified a dimming event that began in late UT 1987 September and ended a few months later in UT 1987 November. From reanalyzing observations from the AAVSO and Weleyan archive, we determined that this event had an ingress timescale of $\sim 30$ days, very similar to what was seen for the 2010-2011 event. The dimming lasted about 50 days and reached a maximum depth of $\sim 1.2 \mathrm{mag}$. Following the same model for these two dimmings as was done for the 2010-2011 and 2012-2013 events and adopting a stellar radius of $1.6 \pm 0.32 R_{\odot}$ (Rodriguez et al. 2013), we estimate the transverse velocity of the occulting bodies to be $\sim 4.6$ 

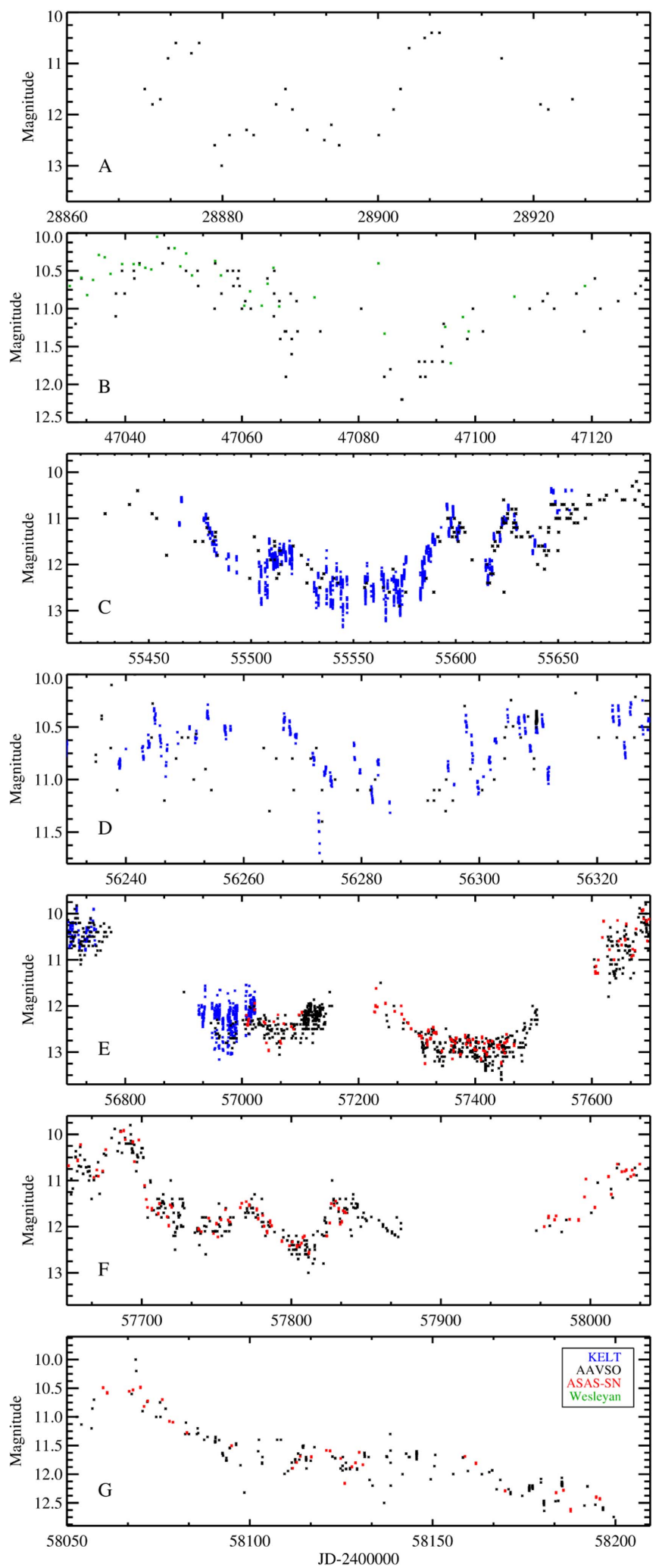

Figure 8. All seven dimmings of RW Aurigae: (A) archival observations showing the 1937-1938 dimming, (B) the 1987-1988 dimming first identified by Berdnikov et al. (2017), (C) recreation of Figure 3 from Rodriguez et al. (2013) showing the 2010-2011 event, (D) recreation of Figure 3 from Rodriguez et al. (2016) showing the 2012-2013 event, and (E) the full 2014-2016 dimming. (F) The full 2016-2017 dimming. (G) The dimming that began in late 2017 .
(1937-1938) and $0.9 \mathrm{~km} \mathrm{~s}^{-1}$ (1987), with both events yielding a similar occulting body width of $\sim 0.08$ and 0.03 au.

\subsection{0-2011, 2012-2013, and 2014-2016 Dimmings$$
\text { "C", " } D \text { ", and " } E \text { " }
$$

From analyzing photometric observations from the KELT survey and the AAVSO, the eclipse in 2010-2011 had a duration of $\sim 180$ days and a depth of $\sim 2$ mag in the optical. A few years later, in 2012-2013, RW Aur experienced a 0.7 mag dimming event for $\sim 40$ days. Using kinematic and geometric arguments, it was determined that the occulting bodies would be moving at $0.9-2.7 \mathrm{~km} \mathrm{~s}^{-1}$, corresponding to a $\sim 0.3$ and $\sim 0.06$ au in width for the 2010-2011 and 2012-2013 events, respectively (Rodriguez et al. 2013, 2016). Unfortunately, these dimmings were not discovered until RW Aur had recovered its nominal brightness, hindering our ability to understand these phenomena through targeted multiband photometric and spectroscopic observations.

The continued monitoring effort of RW Aur by the AAVSO showed that the RW Aur system appeared significantly dimmer after the mid-2014 observing gap (Petrov et al. 2015). Combining these observations with additional data from KELT and the Kutztown University Observatory (KUO), it was determined that the RW Aur system had once again dimmed by $\sim 2$ mag and remained in a dim state through the 2014-2015 observing season (Rodriguez et al. 2016). Spectroscopic observations prior to and during this dimming showed no changes in the emission of $\mathrm{H} \alpha$ or $\mathrm{He}$ I line, suggesting that the high accretion rate for RW Aur A remained constant. Resolved photometric observations of RW Aur showed that RW Aur A was $\sim 3$ mag fainter than the RW Aur B in all optical filters (Lamzin et al. 2017). Additionally, IR photometric monitoring showed a clear decrease in the JHK brightness of the system, but an apparent increase in the $M$ and $L$ filters (Shenavrin et al. 2015). This IR excess was explained by $\sim 1000 \mathrm{~K}$ dust only $0.1-0.2$ au away from RW Aur A and is likely associated with this dimming event. Due to the seasonal observing gap, we are unable to determine precisely when this event began. In 2016 late March, RW Aurigae began to brighten prior to the 2016 observing gap. After the 2016 observing gap, RW Aur was at a brightness of $V \sim 10.8$. Unfortunately, both ingress and egress for this event occurred during seasonal observing gaps, limiting our ability to estimate the transverse velocity of the the occulting body. We estimate a total duration of the 2014-2016 dimming to be $<830$ days, assuming the first point after the 2016 observing gap as the end of the eclipse.

\subsection{Recent Events: 2016-2017 “F," 2017-2018 “G”}

In late 2016 October, after about $\sim 84$ days from the end of the 2014-2016 eclipse, RW Aurigae dimmed by $\sim 2$ mag for the fourth time in less than a decade. This dimming was first identified by Berdnikov et al. (2017). We estimated the ingress to be $\sim 35$ days and the egress to be $\sim 65$ days. Using the same model, this would correspond to a transverse velocity range of $0.4-0.8 \mathrm{~km} \mathrm{~s}^{-1}$. The event lasted until early 2017 October, with a duration of $\sim 335$ days. Similar to what was seen in the 2010-2011 and 2012-2013 dimmings, the 2016-2017 dimming displayed a sinusoidal variation on a similar timescale as the ingress/egress. We estimate the width of the occulting 
Table 2

Results from the Photometric Modeling

\begin{tabular}{|c|c|c|c|c|c|c|c|}
\hline ID & Dimming Event & Ingress/Egress & Transverse Velocity & Duration & Occulter Width & Maximum Depth & Discovery Reference \\
\hline & & (days) & $\mathrm{km} \mathrm{s}^{-1}$ & (days) & (au) & (mag) & \\
\hline A & $1937-1938$ & $\sim 6$ & 4.6 & $\sim 30$ & 0.08 & $\sim 2$ & Berdnikov et al. (2017) \\
\hline B & 1987 & $\sim 30$ & 0.9 & $\sim 50$ & 0.03 & $\sim 1.2$ & Berdnikov et al. (2017) \\
\hline $\mathrm{C}$ & 2010-2011 & $10-30$ & $0.9-2.7$ & $\sim 180$ & $0.1-0.3$ & $\sim 2$ & Rodriguez et al. (2013) \\
\hline $\mathrm{D}$ & 2012-2013 & $\sim 10$ & 2.7 & $\sim 40$ & 0.06 & $\sim 0.7$ & Rodriguez et al. (2016) \\
\hline $\mathrm{E}$ & 2014-2016 & $\cdots$ & $\cdots$ & $<830$ & $\cdots$ & $\sim 2.5$ & Petrov et al. (2015), Rodriguez et al. (2016) \\
\hline $\mathrm{F}$ & 2016-2017 & $35-65$ & $0.4-0.8$ & $\sim 335$ & $0.08-0.15$ & $\sim 2$ & Berdnikov et al. (2017) \\
\hline G & 2017-? & $\geqslant 25$ & $\cdots$ & $>120$ & $\cdots$ & $\geqslant 2$ & This Work \\
\hline
\end{tabular}

Note. The method for estimating the kinematics of the occulting body is described in Section 4 .

body to be $\sim 0.08-0.15 \mathrm{au}$. The longer estimated egress timescale may indicate that the occulting body has a sharp leading edge but a diffuse, more extended trailing edge.

After RW Aurigae recovered from the 2016-2017 dimming, it only stayed at its nominal brightness for $\sim 82$ days before beginning a fifth dimming event in less than a decade. Since the event is ongoing, we only have a small amount of photometric coverage. The dimming event has been going on for $>120$ days. Although it appears that RW Aur is still dimming, it is possible that the ingress is $\sim 25$ days, beginning at 2458067 and ending on 2458093. This would suggest a similar ingress timescale as the 2013 dimming event. To date, this dimming has reached a depth of $\sim 2 \mathrm{mag}$, but the system may continue to fade.

\section{Discussion}

\subsection{Summary of More than a Century of Photometric Observations}

Based on analyzing over 100 years of photometric observations, seven separate dimming events have been identified. It is likely that the eccentric flyby of RW Aur B that disrupted the disk around RW Aur A has significantly influenced the circumstellar environment that contributed to the observed dimming events. From applying a simple model and analyzing each event independently, we estimate physical parameters of the occulting screens, which are shown in Table 2. For each event, we derive a transverse velocity in the range of $0.4-4.6 \mathrm{~km} \mathrm{~s}^{-1}$. Using the 1937-1938 event (which has the fastest estimated velocity) and a $1.4 M_{\odot}$ for RW Aur A, this would correspond to a semimajor axis of $\sim 55$ au, assuming Keplerian motion. This would place the occulting body outside the radius of the dust $(\sim 21$ au, see Section 3.1) and gas ( $\sim 58$ au, see Section 3.2) disks, where Keplerian rotation speeds would be $8 \mathrm{~km} \mathrm{~s}^{-1}$ and $4.7 \mathrm{~km} \mathrm{~s}^{-1}$, respectively (around a $1.4 M_{\odot}$ star). This may suggest that the occulting material causing this event may not be at the same orbital distance as the material that has caused the more recent events.

One change to our analysis that could allow the occulting body to be in orbit within the short truncated disk around RW Aur A would be to have an inclined leading edge relative to the direction of motion (see Figure 9 from Rodriguez et al. 2015). This model allows one to increase the occulting body's velocity, placing it closer to the host star, assuming Keplerian motion. In addition, this inclined edge geometry is what one would expect for the shape of a warp within the disk. Using the 1937-1938 event, which had the shortest ingress timescale (and thus the highest estimated velocity), it would take a leading edge angle of $60^{\circ} .5$ to place the occulting body at the edge of the dust disk. For the 2016-2017 event, which had the longest ingress/egress timescale, it would require a leading edge angle of 4.5 .

We also note that the disk radius in the millimeter range is dominated by large grains; hence, smaller dust grains could still be present up to the edge of the gas disk (at $58 \mathrm{au}$ ), and farther out in stripped material off the disk plane. We note that the estimated velocity derived from each dimming event is consistent with the relative velocities of the observed disrupted gas (see Figure 2). Given the evidence of a star-disk interaction, which has significantly disrupted the circumstellar environment around RW Aurigae, we are not able to place any additional constraints on the location of the occulting features.

The large range in the duration of the seven dimming events, resulting in a wide range of occulting body sizes, could be explained by multiple dust clumps orbiting RW Aur A in Keplerian motion (Rodriguez et al. 2013). However, the large variations in the occulting body parameters could also easily be explained if the dust screen is caused by disk winds (Petrov et al. 2015) or inner disk perturbation (Facchini et al. 2016). Since the estimated kinematics for the more recent events are similar (a few $\mathrm{km} \mathrm{s}^{-1}$; we note that this is not clear for the 2014-2016 event), it is very likely that they are all caused by the same mechanism. Unfortunately, we are not able to distinguish between the plausible interpretations presented in the beginning of Section 4 .

There is a $\sim 73$-year baseline between the start of the 1937 and the start of the 2010 dimming events. Interestingly, photometric dimmings were observed shortly before and after the 1937 event (Berdnikov et al. 2017). Therefore, it is possible that these dimmings occur periodically, but in clusters of dimming events. However, we see only the one dimming event in the late 1980s where our photometric coverage is complete enough to not have missed additional dimmings. Taking the full baseline between the first and last dimming event ( $\sim 73$ years) and assuming Keplerian motion around a 1.4 $M_{\odot}$ star (RW Aur A), this would correspond to a semimajor axis of $\sim 20 \mathrm{au}$. Using the full 110-year baseline for our photometry (see Figure 7), we are able to search for periodic phenomena that correspond to a spatial scale of $\sim 26$ au. This shows that the long baseline of photometric observations from networks like the AAVSO are beginning to probe the same spatial scales as our ALMA observations. 


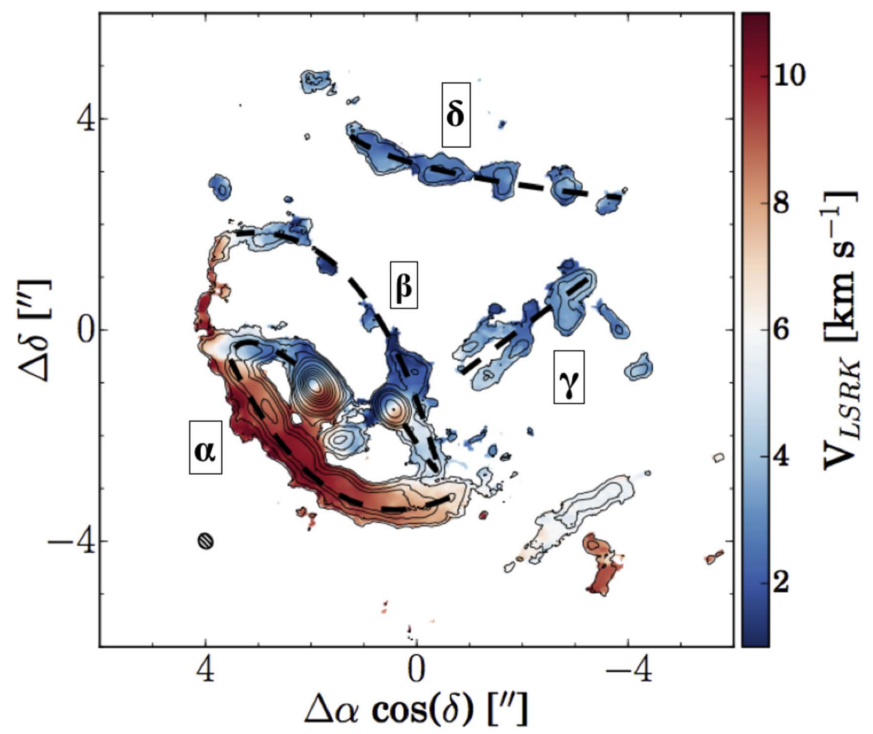

Figure 9. Moment-1 map as shown in Figure 2 overlaid with Moment-0 contours. The original tidal arm discovered by Cabrit et al. (2006) is labeled " $\alpha$," the second counter-spiral arm is labeled " $\beta$ ", and the two new tidal streams identified are labeled " $\delta$ " and " $\gamma$." The dashed black line outlines the two spiral arms that form the apparent ring and the additional tidal streams to the NW.

\subsection{Nature and Morphology of the Tidal Streams}

The presence of additional tidal streams would not be explained with a single star-disk encounter (Clarke \& Pringle 1993; Muñoz et al. 2015). One explanation is that RW Aur B had an initial circumstellar disk prior to the eccentric flyby, and we are seeing the aftermath of a disk-disk collision. Even if RW Aur B had an initial disk, it is unlikely we are seeing the aftermath of one random encounter. The probability for an unbound star-disk flyby would $\sim 10^{-4}$ per star per Myr (see Table 1 of Clarke \& Pringle 1991), making it very unlikely that this is a random encounter. Additionally, the probability is even lower since both RW Aur A and B are known to be pre-main sequence stars (Duchêne et al. 1999), show strong Li I 6707 absorption lines (Stout-Batalha et al. 2000; Takami et al. 2016), and are located near the outer edge of the Taurus-Auriga star formation region. Therefore, we propose that RW Aur A has undergone multiple eccentric flybys $(\geqslant 2)$ from RW Aur B, significantly disrupting the circumstellar material in the system.

From the new high-resolution ALMA observations, we now know that the original tidal arm discovered by Cabrit et al. (2006; “ $\alpha$ ") appears to be part of a projected circumbinary ring of gas around both RW Aur A and B. The observed blueshifted material seen connected to the NW of the RW Aur B disk (" $\beta$ ") is consistent with a second counter-spiral arm to the original tidal arm. Figure 9 outlines the two spirals that would form the projected ring. Therefore, we may be observing the second tidal arm of gas that is connected to the NW extent of the disk around RW Aur B, as suggested from the SPH simulations (see Figure 11 in Dai et al. 2015).

To distinguish between the dual spiral arms and circumbinary ring scenarios, we calculate the escape velocity for the arm and compare this to the observed relative velocity in the ${ }^{12} \mathrm{CO}(2-1)$ Moment-1 map (see Figure 2). We estimate that the arm ranges from 0 !"95-3" (130-420 au) from RW Aur A and 1 ."95-3" (273-420 au) from B. Using a mass of $1.4 M_{\odot}$ and
$0.9 M_{\odot}$ for RW Aur A and B (Ghez et al. 1997), this corresponds to an escape velocity of $2.4-4.4 \mathrm{~km} \mathrm{~s}^{-1}$ and 1.9-2.4 $\mathrm{km} \mathrm{s}^{-1}$ for RW Aur A and B, respectively. From the Moment-1 map, the arm (" $\alpha$ ") is redshifted relative to RW Aur A by up to $6 \mathrm{~km} \mathrm{~s}^{-1}$, with the highest redshift component located in the center of the arm, as seen by Cabrit et al. (2006). This suggests that the arm is unbound at the center and possibly partially bound at the NE and SW extensions, where the arm is closest to either RW Aur A or B. This provides evidence that the apparent ring is likely formed from the original tidal arm connected to the RW Aur A disk and a second counter-spiral arm connected to the RW Aur B disk. We note that the measured redshift and relative distances from RW Aur A and B are lower limits, as we have not corrected for line-of-sight projection effects. A comparison between the calculated escape velocity and observed relative velocity suggests that two spirals are partially unbound and likely expanding outward, as suggested by Cabrit et al. (2006).

In Section 3.2.3, we identified two additional tidal streams " $\gamma$ " and " $\delta$," outlined in Figure 9, to the first discovered tidal arm and its counter-spiral (" $\alpha$ " and " $\beta$ "). The " $\gamma$ " stream extends out $\sim 4^{\prime \prime}$ in the NW direction from RW Aur B. The

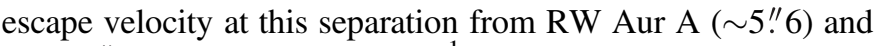
B ( 4."4) is 1.8 and $2 \mathrm{~km} \mathrm{~s}^{-1}$, respectively. The stream is blueshifted by $1-3 \mathrm{~km} \mathrm{~s}^{-1}$ relative to the motion of the entire system, suggesting that some of the tidal stream is unbound. The fourth tidal stream " $\delta$ " in the far NW (see Figure 9) is separated from RW Aur A by 4". 3-6." 3 (602-882 au) and is blueshifted by $2-3 \mathrm{~km} \mathrm{~s}^{-1}$. The estimated escape velocity is $1.7 \mathrm{~km} \mathrm{~s}^{-1}$. Therefore, this fourth tidal stream is likely completely unbound to the system and expanding away. It is not clear at this stage how the morphology of the tidal streams " $\delta$ " and " $\gamma$ " can be reproduced, even with multiple encounters. Further modeling, taking into account an improved orbital solution and a possible secondary disk around RW Aur B prior to the first flyby, are required.

\subsection{Gas Properties in the Tidal Arm and Disks}

To estimate the range of gas temperatures $T_{k}$ and column densities in the CO-emitting region of the tidal arm, we plot the observed ${ }^{12} \mathrm{CO}(3-2) /(2-1)$ ratio as a function of the surface brightness at the line peak in ${ }^{12} \mathrm{CO}(2-1)$ at various positions (see Figure 10) and compare them to simple predictions for an isothermal slab in the Large Velocity Gradient (LVG) approximation, calculated with the online RADEX modeling tool (van der Tak et al. 2007). We assume densities high enough to be in LTE, so as to have only two free parameters left: the gas kinetic temperature $T_{k}$, and the column density per unit velocity, $N(\mathrm{CO}) / d V$. We show in Figure 10 five positions along the arm, including the portion of the arm that is connected to the NE extension of the RW Aur A disk (which we refer to as the "crook"), the three emission peaks along the brightest portion of the arm (near " $\gamma$ "), and the "end" of the arm at $(-1$ !"95, -2 !" 45). For comparison, we also plot the same parameters observed at the line peaks of the A and B disks (both on their blue- and redshifted sides).

We first note that we can readily obtain a lower limit to $T_{k}$ by comparing the ${ }^{12} \mathrm{CO}(2-1)$ surface brightness to the predicted values in the optically thick limit (magenta curve). We obtain $T_{k} \geqslant 20-30 \mathrm{~K}$ in the arm, slightly higher than the $10 \mathrm{~K}$ estimated by Cabrit et al. (2006) from PdBI maps at lower angular resolution, suggesting some degree of clumpiness. In 


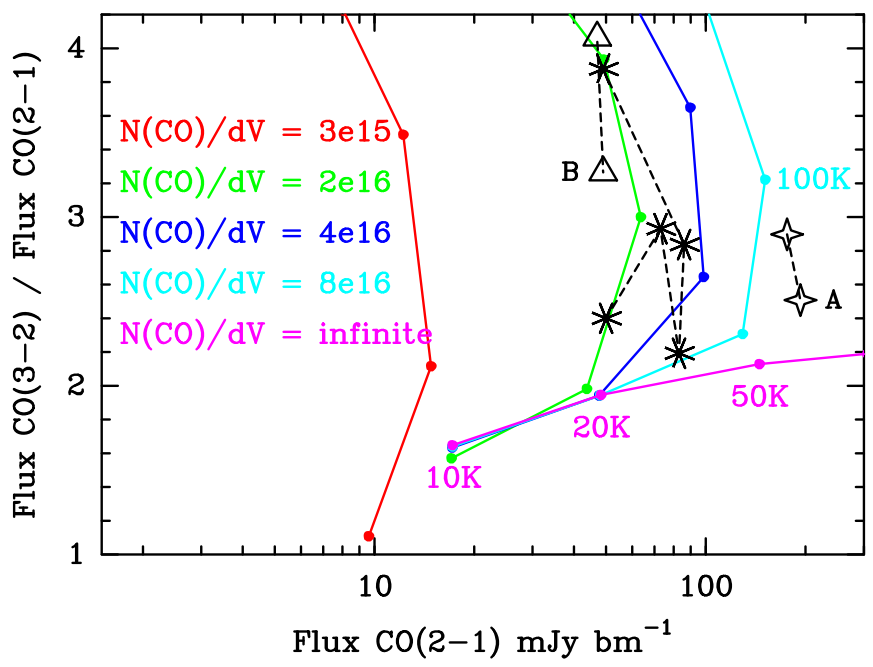

Figure 10. ${ }^{12} \mathrm{CO}(3-2) /{ }^{12} \mathrm{CO}(2-1)$ flux density ratio as a function of the peak line flux density in ${ }^{12} \mathrm{CO}(2-1)$ measured toward the blue and red sides of the RW Aur A disk (connected stars), the blue and red sides of the RW Aur B disk (connected triangles), and five positions along the main tidal arm (connected asterisks, see text). Colored curves show LVG predictions in LTE for an isothermal region with various values of opacity parameter $N(\mathrm{CO}) / d V$ (as labeled, in $\mathrm{cm}^{-2}$ per $\mathrm{km} \mathrm{s}^{-1}$ ) and gas temperatures of $10,20,50$, and $100 \mathrm{~K}$ (dots along each curve) increasing from the lower left corner.

the B disk, we find $T_{k} \geqslant 20 \mathrm{~K}$. We note that any beam-dilution correction would shift the observed points to the right in Figure 10 (the line ratio would be unaffected). Hence these are solid lower limits to the true $T_{k}$. In the A disk, which is likely optically thick in $\mathrm{CO}$ and appears well resolved, we find $T_{k} \simeq 70 \mathrm{~K}$, in excellent agreement with earlier estimates of 60-110 K obtained by Cabrit et al. (2006) from simplified CO line profile modeling.

We also note that the ratio of ${ }^{12} \mathrm{CO}(3-2) /(2-1)$ is always slightly above the predicted value $\simeq 2$ for optically thick isothermal emission, by a factor 1.5-2. The curves in Figure 10 show that in the isothermal assumption, this would require marginally thin emission at higher temperature than in the optically thick limit: assuming negligible beam dilution, $T_{k}$ in the arm would be $100 \mathrm{~K}$ at the crook and 25-50 K elsewhere. An alternative explanation for the ratios $>2$ would be that the emission is optically thick and non-isothermal, with the $\tau=1$ surface of $\mathrm{CO}(3-2)$ probing 1.5-2 times hotter gas than the $\tau=1$ surface of $\mathrm{CO}(2-1)$. This is the most likely explanation for the ratios $\simeq 2.5-4$ observed in the $\mathrm{A}$ and $\mathrm{B}$ disks (Figure 10), since $\mathrm{CO}$ emission remains optically thick out to radii of 100-200 au in typical $\mathrm{T}$ Tauri disks, with $\mathrm{CO}(3-2)$ being thicker than $\mathrm{CO}(2-1)$ and probing higher and warmer atmospheric layers. The same situation may apply to the tidal arm if it has retained some of the internal temperature gradient of the disk after tidal stripping, or if it is externally heated. An upper limit on the $\mathrm{CO}$ optical depth in the arm can be derived from the $3 \sigma$ upper limit in ${ }^{13} \mathrm{CO}(2-1)$ at the peak of the arm $(5.5 \mathrm{mJy} /$ beam in a beam of 0 !' $27 \times 0$." 23$)$; we find a flux density ratio of ${ }^{12} \mathrm{CO}(2-1) /{ }^{13} \mathrm{CO}(2-1)>13$ (after correction for the difference in beam sizes). Assuming similar excitation in the two isotopologues and a solar isotopic abundance ratio of 89 , we infer a ${ }^{12} \mathrm{CO}(2-1)$ optical depth at the peak $<7$. Hence optically thick and non-isothermal CO in the arm at $20-40 \mathrm{~K}$ is not ruled out.

The isothermal, optically thin case still allows us to set a lower limit to the CO column densities, which in the arm would range over $N(\mathrm{CO}) / d V \simeq 2-8 \times 10^{16} \mathrm{~cm}^{-2} /\left(\mathrm{km} \mathrm{s}^{-1}\right)$. Using the determined typical line width in the arm of $2 \mathrm{~km} \mathrm{~s}^{-1}$, we obtain a minimum CO column density of $N_{\mathrm{CO}} \geqslant 4-16 \times 10^{16} \mathrm{~cm}^{-2}$. Since some CO may still be locked in ice mantles, we expect a gas-phase abundance $[\mathrm{CO}] /[\mathrm{H}] \leqslant 10^{-4}$ (the typical interstellar medium value), and infer a lower limit to the hydrogen column density of $N_{\mathrm{H}} \geqslant 0.4-1.6 \times 10^{21} \mathrm{~cm}^{-2}$. Based on this, it appears that a portion of tidal arm passing on the line of sight would contain enough dust to dim the star. We also infer that the arm is a very dense structure. As mentioned in Section 3.2.2, the arm has a typical width of $\simeq 85 \mathrm{au}$. Hence we obtain a lower limit to its (mean) volume density, $n_{\mathrm{H}} \geqslant 3-12 \times 10^{5} \mathrm{~cm}^{-3}$. This confirms a posteriori that LTE should be valid (the critical densities of the $\mathrm{CO}(2-1)$ and (3-2) lines at $\simeq 20 \mathrm{~K}$ are $2 \times 10^{3}$ and $\simeq 7 \times$ $10^{4} \mathrm{~cm}^{-3}$, respectively).

However, we stress that these are only strict lower limits to $N_{\mathrm{H}}$ and $n_{\mathrm{H}}$, as $\mathrm{CO}$ freeze-out at high densities could make a large fraction of the gas invisible. If we were to neglect $\mathrm{CO}$ freeze-out and adopt a standard $\mathrm{CO}$ gas-phase abundance of $10^{-4}$ in the arm, the $\mathrm{CO}(2-1)$ flux integrated over the arm of $3.7 \mathrm{Jy} \mathrm{km} \mathrm{s}{ }^{-1}$ would translate into a total gas mass of $5 \times 10^{-6} M_{\odot}$, where the conversion is for optically thin CO emission in LTE with $T_{k}$ in the range 10-50 K (see Section 3.2.3 in Cabrit et al. 2006). Correcting for a maximum optical depth of $\tau_{12} \leqslant 7$ would increase the mass up to $3.5 \times$ $10^{-5} M_{\odot}$, which is still a very low value. In their analysis of the IM Lup disk, Pinte et al. (2018) found that the gas-phase $\mathrm{CO}$ abundance drops by a factor $10^{-4}$ below $21 \mathrm{~K}$, so that none of the cool disk midplane is detectable. Applying the simple mass formula above to the $\mathrm{CO}(2-1)$ flux of IM Lup (24.7 Jy $\mathrm{km} \mathrm{s}^{-1}$ ), we would infer a disk mass of $2.5 \times 10^{-4} M_{\odot}$, whereas the true disk mass in the illustrative model of Pinte et al. (2018, Appendix B) is $0.6 M_{\odot}$. Because of this, it is not possible to estimate accurately the total gas disk mass from $\mathrm{CO}$ lines alone (Long et al. 2017). The fact that CO excitation temperatures in the tidal arm range down to $\simeq 20 \mathrm{~K}$ in Figure 10, which is close to the freeze-out temperature of $21 \mathrm{~K}$ determined by Pinte et al. (2018), strongly suggests that $\mathrm{CO}$ emission in the arm may also be "freeze-out limited" and suffer from the same problem as in disks.

Keeping these caveats in mind, we note that in the ideal case where (1) $\mathrm{CO}$ ice mantles on dust grains have not been sputtered back into the gas phase during tidal stripping (not unreasonable given the low expected shock speeds $<5 \mathrm{~km} \mathrm{~s}^{-1}$ ), and (2) the structure in density and temperature is not too different between $\mathrm{CO}$-emitting regions in the tidal arm and in the outer disk of A (again not impossible since the CO surface brightness and line ratios are similar in Figure 10), then the ratio of $\mathrm{CO}$ line fluxes between arm and disk would directly yield the mass ratio of gas that was stripped off in the arm compared to that left in the A disk after truncation, without requiring us to know the exact amount of $\mathrm{CO}$ depletion (it would cancel out in the ratio). With integrated flux densities of $3.4 \mathrm{Jy} \mathrm{km} \mathrm{s}{ }^{-1}$ in the A disk and $3.7 \mathrm{Jy} \mathrm{km} \mathrm{s}^{-1}$ over the arm, this ratio would turn out to be close to 1 , and the mass of the ejected material would then be $\sim 50 \%$ of the initial mass of the disk. The tidal encounter would thus have been able to remove a large amount of the disk mass, and likely most of the angular momentum. Interestingly, Muñoz et al. (2015) have shown that when the pericenter of a star-disk encounter is comparable to the disk radius, the gravitational interaction can extract a significant amount of the angular momentum of the disk, thus 
favoring stellar capture, provided that the disk is massive. If the two stars are bound, the undergoing tidal encounter would then have likely removed orbital energy from the system, making the system more bound, and reducing the orbital period. However, we stress that the ejected mass ratio of $50 \%$ derived above is obtained under a very restrictive set of assumptions, and is very uncertain. Numerical simulations of the encounter with improved orbital parameters are necessary to clarify the past and future orbital evolution of the system.

\subsection{Tidal Truncation of the Disks}

The apparently complete encirclement of RW Aur by ejected material suggests that the system has been subject to more than one encounter-i.e., it is a bona fide binary, rather than a oneoff encounter. To estimate the tidal truncation of the primary by the secondary (and vice versa) in this case, we follow Pichardo et al. (2005) and Harris et al. (2012), where the truncation radius of the primary is

$$
R_{t} \approx 0.337\left[\frac{(1-e)^{1.2} \psi^{2 / 3} \mu^{0.07}}{0.6 \psi^{2 / 3}+\ln \left(1+\psi^{1 / 3}\right)}\right] \mathcal{F} a_{p}
$$

and $e, \psi, \mu$, and $a_{p}$ are the eccentricity, mass ratio of the primary star to companion $\left(\psi=M_{p} / M_{s}=1 / q\right)$, the mass fraction of the stellar pair $(\mu=q /[1+q])$, and the projected separation, respectively. $\mathcal{F}$ is the ratio of semimajor axis to projected separation, which Torres (1999) showed to be

$$
\mathcal{F}=\frac{a}{a_{p}}=\frac{1}{1-e \cos (E)} \frac{1}{\sqrt{1-\sin ^{2}(\omega+\nu) \sin ^{2} i}},
$$

where $a, \omega, \nu, E$, and $i$ are the actual separation, longitude of periastron, true anomaly, eccentric anomaly, and inclination, respectively. Inverting the stellar mass ratio gives the truncation of the secondary disk.

Given the unknowns in the above equations, we follow Torres (1999) and Harris et al. (2012) and use a Monte Carlo approach to construct a probability distribution for $R_{t}$. That is, we set up 1000 bins in radius, spanning 0-400 au. We then perform 10 million random samplings of the unknown orbital parameters and place the resulting $R_{t}$ in the appropriate bin for each random realization. Normalizing the resulting binned distribution gives the probability $P\left(R_{t}\right)$.

The randomly sampled parameters are as follows. The longitude of periastron is sampled uniformly from 0 to $2 \pi$ and eccentricity from 0 to some upper limit (we explore both 1 and 0.7). The inclination has a sinusoidal dependence and is therefore sampled evenly in $\operatorname{asin}(r)$ for random variable $r$ in the range 0:1. Last, we require random eccentric and true anomalies. The mean anomaly $M$ is constant with orbital phase and so is randomly sampled with uniform probability from 0 to $2 \pi$. This is related to the eccentric anomaly by

$$
M=E-e \sin (E),
$$

from which we solve for $E$ by bisection starting from $E=\pi$ in the range of 0 to $2 \pi$. The true anomaly is then

$$
\nu=2 \operatorname{atan}\left[\sqrt{\frac{1+e}{1-e}} \tan \left(\frac{E}{2}\right)\right] .
$$

For the current observed separation of 1 " $468 \pm 0$ ". 056 , distance of $140 \mathrm{pc}$, and possible stellar masses of 1.3-1.4 and $0.3-1.0 \mathrm{M}_{\odot}$ for the primary and secondary, respectively, the truncation radius probability distribution is shown in Figure 11. The upper panel is for the primary disk with a maximum eccentricity of 1 and the middle panel for a maximum eccentricity of 0.7 . The lower panel is for the secondary disk with a maximum eccentricity of 0.7 . The multi-valued nature of the distribution comes from the spread in possible stellar mass ratios.

Both the 58 and 38 au observed gas radii of the primary and secondary disks (Table 1) are consistent with the most probable tidal truncation radii in Figure 11 for an eccentricity of 0.7 (although note that we did not constrain the eccentricity through this approach). Although evidence of interaction is obvious in RW Aur in the form of ejected material, this provides further evidence that the disk extents are being set by the gravitational interaction of the binary pair. Furthermore, since an eccentricity $<1$ gives a peak in the distribution consistent with the observed disk radius, this is further evidence that the system is bound and has undergone several interactions.

\section{Conclusions}

The main results from our analysis of the new ALMA observations of RW Aur combined with $\sim 100$ years of photometric observations are the following:

1. Both RW Aur A and B show clear evidence of Keplerian motion in the disks around each star. RW Aur A is $\sim 8$ times brighter in the millimeter continuum than RW Aur B. The two disks are compact (15-20 au) and misaligned to each other by $12^{\circ}$ or $57^{\circ}$.

2. The tidal arm discovered by Cabrit et al. (2006) is part of an apparent circumbinary ring of gas. However, our analysis suggests that the ring-like geometry is a projection effect and is created by the tidal arm around RW Aur A combined with a counter-spiral arm around RW Aur B, as predicted in SPH simulations (Dai et al. 2015). The original tidal stream is at least partially unbound and likely expanding outward from RW Aur A.

3. Photometric dimming events have been occurring for $>80$ years, with the first event in 1937-1938 and the most recent event still ongoing. We have identified seven different dimmings, each varying in depth and duration. Long-baseline photometry is beginning to explore the same spatial scales as ALMA.

4. The gas extents of the primary and secondary disks (58 and $38 \mathrm{au}$, respectively) are entirely consistent with the most probable values expected to result from tidal truncation.

5. RW Aur B has likely undergone multiple eccentric flybys of RW Aur A, as evident by the multiple tidal streams observed.

Future observations of RW Aur should try to understand the influence that these star-disk interactions had on the observed high accretion rate of RW Aur A. Additionally, the observed occultation events may suggest that circumstellar material in RW Aur is coalescing to form larger structures. Therefore, it is possible that we are observing the early stages of planet formation. However, it is not clear what potential these small truncated circumstellar disks will have for future planet formation. Higher angular resolution millimeter mapping of the RW Aur A and B disks may provide additional, more conclusive evidence of ongoing planet formation through the 

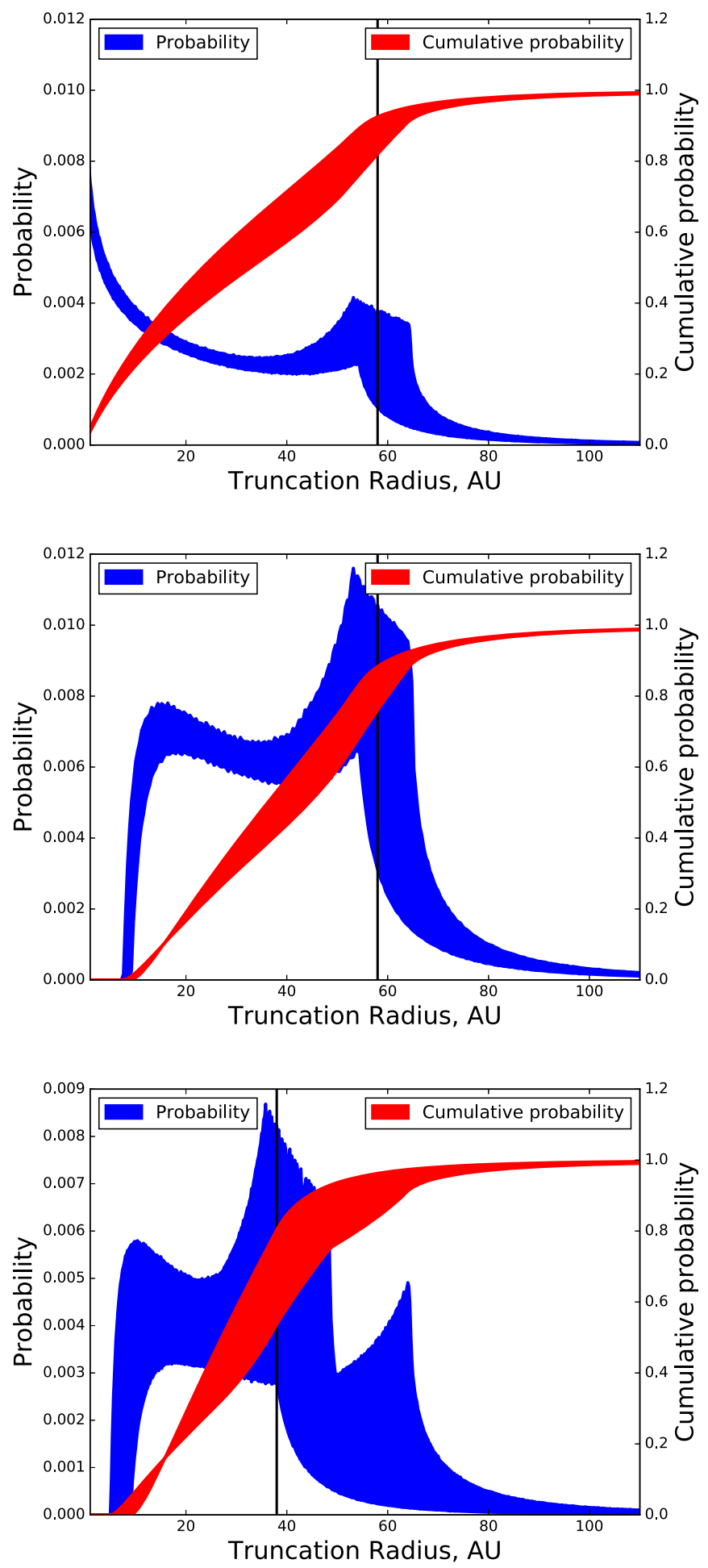

Figure 11. (Cumulative) probability distribution of the truncation radius of the RW Aur disks. The multi-valued nature of the distributions at any given truncation radius is due to the uncertainty in the stellar masses. The upper and middle panels are the distributions for the primary disk, with a maximum eccetricity of 1 and 0.7 , respectively. The lower panel is the probability distribution for the secondary disk for a maximum eccentricity of 0.7 . The black vertical line in each case is the current observed extent of the disk.

presence of gaps in their disks. Our new ALMA observations show multiple tidal streams of gas, which suggests that we are seeing the aftermath of multiple flyby interactions that have significantly influenced the circumstellar environment in RW
Aur. New simulations should try to replicate the observed gas structure and kinematics by allowing for multiple star-disk interactions. Additionally, these simulations should explore the possibility that RW Aur B had an initial disk prior to its first close encounter with RW Aur A.

Work performed by J.E.R. was supported by the Harvard Future Faculty Leaders Postdoctoral fellowship. R.A.L. gratefully acknowledges funding from NRAO Student Observing Support. T.J.H. is funded by an Imperial College London Junior Research Fellowship. S.C. and J.P. acknowledge support from the Programme National "Physique et Chimie du Milieu Interstellaire" (PCMI) of CNRS/INSU with INC/INP co-funded by CEA and CNES.

This paper makes use of the following ALMA data: ADS/JAO. ALMA\#2015.1.01506.S and ADS/JAO.ALMA\#2016.1.00877. S. ALMA is a partnership of ESO (representing its member states), NSF (USA) and NINS (Japan), together with NRC (Canada), MOST and ASIAA (Taiwan), and KASI (Republic of Korea), in cooperation with the Republic of Chile. The Joint ALMA Observatory is operated by ESO, auI/NRAO and NAOJ.

We acknowledge with thanks the variable star observations from the AAVSO International Database contributed by observers worldwide and used in this research.

\section{ORCID iDs}

Joseph E. Rodriguez (iD https://orcid.org/0000-00018812-0565

Ryan Loomis (i) https://orcid.org/0000-0002-8932-1219

Sylvie Cabrit (iD https://orcid.org/0000-0002-1593-3693

Stefano Facchini (iD https://orcid.org/0000-0003-4689-2684

Eric L. N. Jensen (i) https://orcid.org/0000-0002-4625-7333

Keivan G. Stassun (ib https://orcid.org/0000-0002-3481-9052

Jérôme Pety (iD https://orcid.org/0000-0003-3061-6546

\section{References}

Andrews, S. M., \& Williams, J. P. 2005, ApJ, 631, 1134

Antipin, S., Belinski, A., Cherepashchuk, A., et al. 2015, IBVS, 6126, 1

Artymowicz, P., \& Lubow, S. H. 1994, ApJ, 421, 651

Beck, T. L., \& Simon, M. 2001, AJ, 122, 413

Beckwith, S. V. W., \& Sargent, A. I. 1993, ApJ, 402, 280

Berdnikov, L. N., Burlak, M. A., Vozyakova, O. V., et al. 2017, AstBu, 72, 277

Bisikalo, D. V., Dodin, A. V., Kaigorodov, P. V., et al. 2012, ARep, 56, 686 Bouvier, J., Grankin, K., Ellerbroek, L. E., Bouy, H., \& Barrado, D. 2013, A\&A, 557, A77

Bozhinova, I., Scholz, A., Costigan, G., et al. 2016, MNRAS, 463, 4459

Cabrit, S., Pety, J., Pesenti, N., \& Dougados, C. 2006, A\&A, 452, 897

Chou, M.-Y., Takami, M., Manset, N., et al. 2013, AJ, 145, 108

Clarke, C. J., \& Pringle, J. E. 1991, MNRAS, 249, 584

Clarke, C. J., \& Pringle, J. E. 1993, MNRAS, 261, 190

Correia, S., Zinnecker, H., Ratzka, T., \& Sterzik, M. F. 2006, A\&A, 459, 909 Csépány, G., van den Ancker, M., Ábrahám, P., et al. 2017, A\&A, 603, A74 Dai, F., Facchini, S., Clarke, C. J., \& Haworth, T. J. 2015, MNRAS, 449, 1996 Dougados, C., Cabrit, S., Lavalley, C., \& Ménard, F. 2000, A\&A, 357, L61 Duchêne, G., Monin, J.-L., Bouvier, J., \& Ménard, F. 1999, A\&A, 351, 954 Facchini, S., Manara, C. F., Schneider, P. C., et al. 2016, A\&A, 596, A38 Foreman-Mackey, D., Hogg, D. W., Lang, D., \& Goodman, J. 2013, PASP, 125,306

Gaia Collaboration, Brown, A. G. A, Vallenari, A., et al. 2016, A\&A, 595, A2 Ghez, A. M., Neugebauer, G., \& Matthews, K. 1993, AJ, 106, 2005

Ghez, A. M., White, R. J., \& Simon, M. 1997, ApJ, 490, 353

Harries, T. J. 2000, MNRAS, 315, 722

Harris, R. J., Andrews, S. M., Wilner, D. J., \& Kraus, A. L. 2012, ApJ, 751,115

Hartigan, P., Edwards, S., \& Ghandour, L. 1995, ApJ, 452, 736 
Herbig, G. H., \& Bell, K. R. 1988, Third Catalog of Emission-Line Stars of the Orion Population, Vol. 3 (Santa Cruz, CA: Lick Observatory)

Herbst, W., Herbst, D. K., Grossman, E. J., \& Weinstein, D. 1994, AJ, 108, 1906

Ida, S., Larwood, J., \& Burkert, A. 2000, ApJ, 528, 351

Jensen, E. L. N., \& Akeson, R. 2014, Natur, 511, 567

Kochanek, C. S., Shappee, B. J., Stanek, K. Z., et al. 2017, PASP, 129, 104502

Lamzin, S., Cheryasov, D., Chuntonov, G., et al. 2017, in ASP Conf. Ser. 510, Stars: From Collapse to Collapse, ed. Y. Y. Balega et al. (San Francisco, CA: ASP), 356

Lee, C.-F., Li, Z.-Y., Ho, P. T. P., et al. 2017, ApJ, 843, 27

Leinert, C., Zinnecker, H., Weitzel, N., et al. 1993, A\&A, 278, 129

Long, F., Herczeg, G. J., Pascucci, I., et al. 2017, ApJ, 844, 99

Loomis, R. A., Öberg, K. I., Andrews, S. M., et al. 2018, AJ, 155, 182

Loomis, R. A., Öberg, K. I., Andrews, S. M., \& MacGregor, M. A. 2017, ApJ, 840,23

López-Martín, L., Cabrit, S., \& Dougados, C. 2003, A\&A, 405, L1

McMullin, J. P., Waters, B., Schiebel, D., Young, W., \& Golap, K. 2007, in ASP Conf. Ser. 376, Astronomical Data Analysis Software and Systems XVI, ed. R. A. Shaw, F. Hill, \& D. J. Bell (San Francisco, CA: ASP), 127 Mundt, R., \& Eislöffel, J. 1998, AJ, 116, 860

Muñoz, D. J., Kratter, K., Vogelsberger, M., Hernquist, L., \& Springel, V. 2015, MNRAS, 446, 2010
Osterloh, M., \& Beckwith, S. V. W. 1995, ApJ, 439, 288

Osterloh, M., Thommes, E., \& Kania, U. 1996, A\&AS, 120, 267

Petrov, P. P., Gahm, G. F., Djupvik, A. A., et al. 2015, A\&A, 577, A73

Pichardo, B., Sparke, L. S., \& Aguilar, L. A. 2005, MNRAS, 359, 521

Pinte, C., Ménard, F., Duchêne, G., et al. 2018, A\&A, 609, A47

Richichi, A., Leinert, C., Jameson, R., \& Zinnecker, H. 1994, A\&A, 287, 145

Rodriguez, J. E., Pepper, J., Stassun, K. G., et al. 2013, AJ, 146, 112

Rodriguez, J. E., Pepper, J., Stassun, K. G., et al. 2015, AJ, 150, 32

Rodriguez, J. E., Reed, P. A., Siverd, R. J., et al. 2016, AJ, 151, 29

Rundle, D., Harries, T. J., Acreman, D. M., \& Bate, M. R. 2010, MNRAS, 407, 986

Schneider, P. C., Günther, H. M., Robrade, J., et al. 2015, A\&A, 584, L9

Shappee, B. J., Prieto, J. L., Grupe, D., et al. 2014, ApJ, 788, 48

Shenavrin, V. I., Petrov, P. P., \& Grankin, K. N. 2015, IBVS, 6143, 1

Simon, M., Ghez, A. M., Leinert, C., et al. 1995, ApJ, 443, 625

Skinner, S. L., \& Güdel, M. 2014, ApJ, 788, 101

Stout-Batalha, N. M., Batalha, C. C., \& Basri, G. S. 2000, ApJ, 532, 474

Takami, M., Wei, Y.-J., Chou, M.-Y., et al. 2016, ApJ, 820, 139

Torres, G. 1999, PASP, 111, 169

Trilling, D. E., Stansberry, J. A., Stapelfeldt, K. R., et al. 2007, ApJ, 658, 1289

van der Tak, F. F. S., Black, J. H., Schöier, F. L., Jansen, D. J., \& van Dishoeck, E. F. 2007, A\&A, 468, 627

van Leeuwen, F. 2007, A\&A, 474, 653

Woitas, J., Leinert, C., \& Köhler, R. 2001, A\&A, 376, 982 